$\begin{array}{ll}\text { Italique } & \text { Italique } \\ \text { Poésie italienne de la Renaissance }\end{array}$

XII | 2009

Varia

\title{
La presenza di Luigi Groto in Shakespeare e negli autori elisabettiani
}

\section{Barbara Spaggiari}

\section{OpenEdition}

\section{Journals}

\section{Edizione digitale}

URL: http://journals.openedition.org/italique/232

DOI: 10.4000/italique.232

ISSN: 1663-4438

\section{Editore}

Librairie Droz

\section{Edizione cartacea}

Data di pubblicazione: 1 novembre 2009

Paginazione: 173-198

ISSN: 1423-3983

\section{Notizia bibliografica digitale}

Barbara Spaggiari, «La presenza di Luigi Groto in Shakespeare e negli autori elisabettiani », Italique

[Online], XII | 2009, online dal 01 novembre 2012, consultato il 19 avril 2019. URL : http:// journals.openedition.org/italique/232 ; DOI : 10.4000/italique.232 


$$
\text { B ARBARA SPAGGIARI }
$$

LA PRESENZA DI LUIGI GROTO

I N S H A KES P E A R E

E NEGLI A UTORI ELISABETTIA N I 



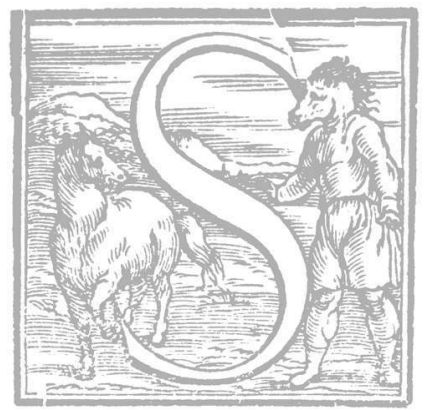

Econdo una prassi consolidata nell'ambito della filologia inglese, ogni edirione di un'opera teatrale di Shakespeare è accompagnata da un apposito capitolo sulle fonti. Intitolato semplicemente Sources, ${ }^{\mathrm{I}}$ questo capitolo riassume in forma ora stringata, ora più esauriente e distesa, le indicazioni che si sono venute accumulando nei commenti precedenti, nel corso di una stratificazione spesso secolare.

Nel caso di Romeo and Juliet le edizioni di riferimento, prima fra tutte quella di Brian Gibbons per "The Arden Shakespeare », ${ }^{2}$ condividono un certo numero di tratti caratteristici, purtroppo non tutti positivi, uno dei quali è l'assenza della distinzione fondamentale fra archetipo, ${ }^{3}$ modello mediato ${ }^{4} e$ modello diretto. ${ }^{5}$ Un altro è la tendenza a mettere sullo stesso piano, ai fini attributivi, elementi macroscopici come la trama principale (plot) o secondaria (sub-plot) di un dramma, con certi particolari sviluppi della narrazione (episode or event) o con il rilievo dato a singoli personaggi (characters); senza poi parlare di altri dettagli la cui pertinenza può essere valutata solo in un quadro più generale (scattered details). ${ }^{6}$

Nessun dubbio sul fatto che «the story was well established in the Is80s and Shakespeare may have known it for a number of years before I59I, in more than one version, before he decided to dramatize it $\gg{ }^{7}$ Fra le differenti versioni della storia di Giulietta e Romeo, quattro si impongono come precedenti ineludibili della tradizione quale si viene stabilendo nel corso del Cinquecento: l'Istoria novellamente ritrovata di due nobili amanti di Luigi Da Porto; ${ }^{8}$ la novella già citata del Bandello, che a Da Porto dichiaratamente si ispira; 9 il poemetto in ottava rima di Gherardo Boldieri; ${ }^{1 \circ}$ e finalmente la Hadriana di Luigi Groto.

Composta quando l'autore aveva circa vent'anni, fra il is6o e il I56I, ma pubblicata solo nel I 578 (Venezia, Farri), la Hadriana costituisce uno dei più alti esempi di tragedia manierista: per la programmatica frattura rispetto alla norma aristotelica; per l'uso oltranzista di un linguaggio metaforico, iperbolico, ossimorico, che mette a contribuzione tutto l'ampio repertorio di figure retoriche caratterizzante anche le Rime grotiane; per l'alternarsi di momenti visionari e allucinati con scene di gusto sepolcrale e orrifico. Il ritmo spesso martellante dell'endecasillabo sdrucciolo scandisce, a colpi di anafore o di antitesi, i passaggi più sensibili di una vicenda che veste il macabro di un'accesa sensualità.

Le dieci stampe che si succedettero dal 1578 al $1626^{11}$ dimostrano che questa tragedia ebbe un grande successo editoriale, dunque di pubblico, superando tutti gli altri componimenti teatrali del Groto, ${ }^{\mathrm{I} 2}$ anche quelli che, come il 
Pentimento amoroso, segnarono una tappa importante, e non contestata, nell'evoluzione del dramma pastorale in Francia e nell'Inghilterra elisabettiana. $^{\mathrm{I}} 3$

Quando Shakespeare scrive Romeo and Juliet fra il 1593 e il $1596,{ }^{\mathrm{I} 4}$ la Hadriana ha già avuto quattro edizioni, e la fama del Cieco d'Adria è ben consolidata in Inghilterra, dove permarrà intatta fino almeno agli inizi del secolo successivo. Lo dimostrano, al di là di ogni ragionevole dubbio, almeno tre fatti acquisiti:

I. in Volpone di Ben Jonson (I605), "s Lady Would-Be elenca le presunte competenze che dovrebbero fare di lei una donna di classe, colta e raffinata. Afferma di dedicare ogni giorno alcune ore alla musica e alla pittura, perché una vera signora deve conoscere le lettere e le arti, saper parlare, scrivere, dipingere. Cita a sproposito Platone e Pitagora, attirandosi la risposta sprezzante di Volpone ("your bighest female grace is silence»). Nient'affatto scoraggiata, chiede chi siano i poeti preferiti dal suo interlocutore, asserendo in anticipo di conoscerli tutti per averli già letti:

Which o' your Poets? Petrarch? or Tasso? or Dante?

Guarini? Ariosto? Aretine?

Cieco di Hadria? I have read them all.

$$
\text { (III. } 4,79-8 \mathrm{I})^{\mathrm{I} 6}
$$

Non è certo priva d'interesse questa breve hit-parade degli scrittori italiani la cui frequentazione, sullo scorcio del XVI secolo, era ritenuta indispensabile: ovviamente i classici Dante e Petrarca (non Boccaccio), poi l'Ariosto e l'Aretino per la prima metà del Cinquecento, Tasso e Groto per la seconda metà. Si

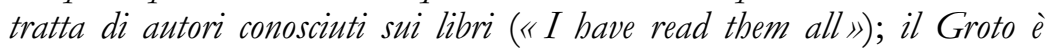
addirittura citato attraverso il suo nom de plume ("Cieco di Hadria»), sebbene questo dovesse risultare non poco ostico alla pronuncia inglese. ${ }^{17}$

2. un'intera serie di epigrammi tradotti, senza citarlo, dal Groto si trova nella Poetical Rhapsody di Francis Davison (I602). ${ }^{18}$ Il riconoscimento della fonte grotiana risale alla fine del XIX secolo, come informa il classico studio di Hutton, Anthology in Italy: "Noticed by J. M. Thomson in Bullen's edition $\gg .{ }^{\text {I9 }}$

3. un altro imitatore del Groto fu William Drummond of Hawthornden (I585-I649), soprannominato il Petrarca scozzese, autore di una raccolta di Poems (I6IG) di vario argomento e metro, come informa il titolo della princeps: "Amorous, Funerall, Divine, Pastorall, in Sonnets, Songs, Sextains, Madrigals ». ${ }^{20}$ La sezione intitolata Madrigals and epigrammes 
giustifica di per sé l'interesse di Drummond per le liriche del Groto. ${ }^{21}$ Due sono però $i$ motivi che, oltre alle imitazioni puntuali, ${ }^{22}$ fanno di Drummond una tessera importante nella nostra ricostruzione storica. Il primo riguarda la ricca biblioteca personale, circa cinquecento volumi, di cui egli fece dono, ancora in vita, al Tounis College (oggi University of Edimburgh) dove aveva compiuto gli studi; ${ }^{23}$ vi si trovano quattro opere del Groto, tre originali e una tradotta: "Rime del Luigi Groto cieco d'Adria [It.I9]: $n^{\circ}$ I229 GROTO, Luigi. Delle rime... ", la Dalida (ed. I609: $n^{\circ}$ I228), Il thesoro (ed. I599: $n^{o}$ I230), infine Le repentir d'amour de Diéromène, pastorale imitée de l'italien de L.G.C.D.H.i par R[olland] B[risset] G[entilhomme] T[ourangeau] $\left(n^{\circ} 1058\right){ }^{24}$

Il secondo motivo d'interesse risiede nelle liste, conservate con grande scrupolo nei manoscritti di Drummond, dei libri studiati e letti al di fuori di quelli personalmente posseduti. ${ }^{25}$ Dall'Appendix I: Books red be me, risulta che nell'anno I6I2 lesse le "Rime di Luigi Groto cieco d'adria ", probabilmente nell'edizione in tre parti del I6ro. ${ }^{26}$

Un'ulteriore affinità del Drummond con Groto si ricava dal possesso di quattro opere di Bernardino Ochino: Il catechismo, Basel, I56r; Disputa...intorno alla presenza del corpo di Giesu Christo nel sacramento della cena, Basel, Is6r; Prediche (Genève, I542?); di nuovo Prediche, Basel I569. ${ }^{27}$

Da quanto detto finora a proposito di Ben Jonson, Francis Davison e William Drummond, si raccoglie la certezza che sullo scorcio del Cinquecento il Cieco d'Adria, con buona pace dei suoi detrattori antichi e moderni, era per gli intellettuali inglesi una figura di spicco della letteratura italiana, nell'ambito sia della drammaturgia che della poesia lirica. ${ }^{28}$

Ulteriori informazioni fornite dalle testimonianze coeve a Shakespeare riguardano $i$ modi della trasmissione delle opere del Groto che avveniva, ovviamente, attraverso le stampe.

A questo proposito, risulta una singolare convergenza fra chi sostiene l'influenza grotiana sul Romeo and Juliet, ${ }^{29}$ e chi invece virulentemente la nega: sia gli uni che gli altri insistono sull'impossibilità che Shakespeare abbia potuto conoscere la Hadriana. Certo non l'ha vista rappresentata su un improbabile palcoscenico inglese, e ancor meno polesano; ma le tragedie, nel Cinquecento e prima degli elisabettiani, erano fatte per essere lette ancor più che per essere portate sulla scena. ${ }^{30} \mathrm{Ne}$ abbiamo ora la prova diretta. La circolazione dell'Hadriana in Inghilterra, nonché la circostanza e il luogo in cui Shakespeare ha potuto aver accesso alla tragedia grotiana, sono ormai stabiliti grazie alla pur cauta segnalazione di Giulietta Bazoli. ${ }^{3}$ 
Già il classico studio di David Orr aveva messo in luce l'importanza delle biblioteche private come veicolo di conoscenza dei testi italiani nell'Inghilterra elisabettiana, facendo esplicito riferimento a quella di John Florio. ${ }^{32}$ Vale la pena di esaminare $i$ dettagli della questione.

Fra il I592 e il I594, Shakespeare si trovava di fatto alle dipendenze del terzo conte di Southampton, Henry Wriothesle, uno dei grandi mecenati dell'epoca, cui il giovane talento dedicò Venus and Adonis (I593), The Rape of Lucrece (I594) e il libro dei Sonnets che sarebbe stato pubblicato solo nel I609. ${ }^{33}$ In quegli stessi anni e per lo stesso conte, Giovanni (John) Florio ${ }^{34}$ attendeva alla compilazione del primo dizionario italiano-inglese, ${ }^{35}$ per rispondere alla crescente esigenza, da parte dell'aristocrazia anglosassone, di avvicinarsi senza intermediari agli originali della letteratura dominante nel Rinascimento. ${ }^{36}$

Il dizionario approntato dal Florio include, nella seconda edizione, una lista di opere da lui consultate che occupa tre pagine non numerate all'inizio del grande volume in-folio, esattamente fra le poesie in lode dell'autore ${ }^{37}$ e il suo ritratto: ${ }^{8}$ The names of the Authors and Books that have been read of purpose for the collecting of this Dictionarie. Fra $i$ titoli, seguiti dal nome dell'autore, e disposti in ordine alfabetico su due colonne (a-f), appaiono ben otto opere del Groto:

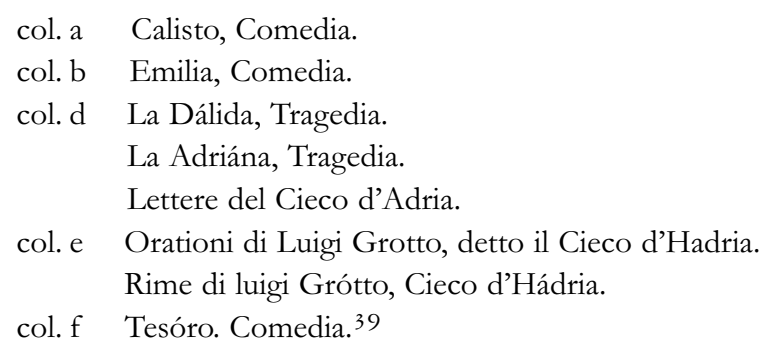

Nell'elenco manca solo il Pentimento amoroso per completare la scelta di quanto di meglio ha lasciato Luigi Groto nella sua instancabile attività di prosatore (le Lettere famigliari e le Orazioni volgari), drammaturgo (le due tragedie e tre delle commedie) e lirico (le Rime, forse usufruite nell'ed. del I6Io). Per tornare alle fonti del Romeo and Juliet, oltre alla Hadriana il Florio disponeva delle "Novelle del Bandello, volumi tre », nell'edizione cioè del I554 (In Lucca: per il Busdrago). Dalla stessa raccolta del Bandello (I 22) deriva anche la trama di Much ado about nothing, mentre agli « Hecatommiti di $M^{r}$ Gio. battista Giráldi Cinthio », listati nella col. c, Shakespeare si è notoriamente ispirato sia per il plot dell'Othello (Hecat. III 7) che per Measure for Measure (Hecat. IV 2). In tutti e tre $i$ casi l'italian connection non è contestata. ${ }^{40}$ 
Tutte le condiz̨ioni sono dunque riunite perché, nella cerchia privilegiata di letterati che gravitava intorno al conte di Southampton, Shakespeare abbia potuto conoscere direttamente il testo della tragedia grotiana negli anni immediatamente precedenti alla stesura del Romeo and Juliet, con in più la facoltà di avvalersi della mediazione linguistica del Florio. ${ }^{4 \mathrm{I}}$

Una volta verificato come e quando Shakespeare poté aver accesso alla stampa della Hadriana, conviene tornare all'accertamento dei dati attraverso un confronto testuale possibilmente scevro da pregiudizi. Dall'analisi risultano tre luoghi in cui le coincidenze puntuali (close verbal parallels) tra la Hadriana $e$ il Romeo and Juliet sconsigliano, come vedremo, qualunque ipotesi poligenetica. ${ }^{42}$
a. Hadriana, Atto 2, sc. I, vv. 86-94
Lat.
Attenderò, che fuori esca Hadriana,
Poi che a quest'hora sempre esce la
A veder s'io ci son, com'è composto
Tra noi. E par, ch'io senta aprir la porta,
La qual meglio chiamar posso Oriente.
Ecco spunta il mio Sol cinto di nubi
A mezza notte. Mira, come gli astri
Dan loco al lume suo smarriti in vista,
Come stan l'aure a vagheggiarlo intente. notte

\section{b. Hadriana, Atto 2, sc. 3, vv. 230-54}

Hadr.

Deh, (se mi amate) non partite ancora.

Perchè pensando, che partir dovete,

La mente impari a sofferirlo meglio.

Lat. $[\cdots]$.

E che facciam più qui

E (s'io non erro) è presso il far del giorno.

Udite il Rossignuol che con noi desto,

Con noi geme fra i spini, e la rugiada

Col pianto nostro bagna l'erbe. Ahi lasso.

Rivolgete la faccia a l'Oriente.

Ecco incomincia a spuntar l'alba fuori

Portando un altro Sol sopra la terra,

Che però dal mio Sol resterà vinto.

Hadr.

Ahimè, ch'io gelo. Ahimè, ch'io tremo tutta.

$[\cdots]$
Romeo and Juliet, Act 2, Scene 2, vv. 2-9

[Enter Juliet above]

Romeo

But soft, what light through yonder window breaks?

It is the east and Juliet is the sun!

Arise fair sun and kill the envious moon

Who is already sick and pale with grief

That thou her maid art far more fair than she.

Be not her maid since she is envious, Her vestal livery is but sick and green And none but fools do wear it. Cast it off.

Romeo and Juliet, Act 3, Scene 5, vV. I-I I

Juliet

Wilt thou be gone? It is not yet near day. It was the nithtingale and not the lark

That pierc'd the faerful hollow if thine ear.

Nightly she sings on yond pomegranate tree.

Believe me, love, it was the nightingale.

Romeo

It was the lark, the harald of the morn,

No nightingale. Look, love, what envious streaks

Do lace the severing clouds in yonder east.

Nights candles are burnt out, and jocund day

Stands tiptoe on the misty mountain tops.

I must be gone and live, or stay and die. 
O del mio ben nemica avara notte,

Perchè sì ratto corri, fuggi, voli

A sommerger te stessa, e me nel mare.

Te ne lo Ibero, e me nel mar del pianto?

$\mathrm{O}$ da invidia accelerata aurora,

Che agli altri luce, a me tenebre apporti...

c. Hadriana, Atto 3, sc. 3, vv. I86-202

Mago $[\ldots]$

Io vi darò una polve, che $\mathrm{mi}$ diede

Di sua man propria il Sonno a l'hora, quando

Io visitai le sue cimerie case,

Piena di inestimabile virtute.

Questa beendo voi con l'acqua cruda,

Darà principio a lavorar fra un poco,

$\mathrm{E}$ vi addormenterà sì immota, e fissa,

E d'ogni senso renderà sì priva:

Il calor naturale, il color vivo,

E lo spirar vi torrà sì, sì i polsi

(In cui è il testimonio della vita)

Immobili staran senza dar colpo;

Che alcun per dotto fisico, che sia,

Non potrà giudicarvi altro, che morta.

Et io, che lo saprò, ne starò in dubbio.

E tante hore starete così, quanta

Fia stata la misura de la polve.
Romeo and Juliet, Act 4, Scene I, vv. 93-105

Friar [...]

Take thou this vial, being then in bed,

And the distilling liquor drink thou off;

When presently through all thy veins shall run

A cold and drowsy humour, for no pulse

Shall keep his native progress, but surcease.

No warmth, no breath shall testify thou livest,

The roses in thy lips and cheeks shall fade

To wanny ashes, thy eyes'windows fall

Like death when he shuts up the day of life.

Each part depriv'd of supple government

Shall stiff and stark and cold appear, like death,

And in this borrow'd likeness of shrunk death

Thou shalt continue two and forty hours...

Tenendo conto dellintera tradizione della storia di Giulietta e Romeo, abbiamo potuto evidenziare una serie di novità che sono comuni soltanto a Groto e Shakespeare, e che non trovano riscontro in nessuno dei possibili antecedenti, siano essi latini, italiani, francesi o inglesi:

a. all'inizio dell'incontro notturno dei due protagonisti (Hadriana Atto 2, sc. I = Romeo and Juliet Act 2, sc.2):

- la similitudine della donna amata che entra dalla porta, come da Oriente entra il sole; ${ }^{43}$

b. nel commiato degli amanti all'alba (Hadriana Atto 2, sc.3 = Romeo and Juliet Act 3, sc.5):

- il motivo dell'usignolo; ${ }^{44}$

- la notte definita «nemica » e « avara », l'aurora « invidiosa $»{ }^{45}$ 
c. nell'ultimo atto (Hadriana Atto 3, sc.3 $=$ Romeo and Juliet Act 4, sc.I):

- la descrizione degli effetti della pozione destinata a simulare la morte. ${ }^{46}$

Cominciamo l'analisi da quest'ultimo luogo, dov'è sensibile l'eco dantesca di Inf. I 90 "Vedi la bestia per cu'io mi volsi: / aiutami da lei, famoso saggio, / ch'ella mi fa tremar le vene e i polsi ", locupletata da Inf. I3 63 " tanto ch' $i$ ' ne perdei li sonni e' polsi $\gg{ }^{47}$ La poca vita riecheggia ancora in Petrarca Rvf 328, 5-6 "Qual à già i nervi e i polsi e i penser' egri, / cui domestica febbre assalir deve ", con nervi che rimpiazza vene. ${ }^{48}$ All'interno della tradizione di Giulietta e Romeo, "le vene e i polsi » è sintagma utilizzato solo nella novella del Sermini su Vannino da Perugia. ${ }^{49}$

Le corrispondenze puntuali fra Groto e Shakespeare riguardano i tre sintomi per eccellenza della vita, cioè il calor naturale = warmth; il color vivo = the roses, che accende labbra e guance; $e$ il respiro, lo spirar = breath. Ad essi si aggiunge, percettibile solo al tatto, il battito del cuore, ${ }^{5 \circ}$ il ritmo del sangue che scorre nelle vene ed è testimonianza di vita (in cui è il testimonio della vita $=$ shall testify thou livest) anche quando gli altri tre elementi scompaiono, quando cioè il calore lascia il posto al freddo, il colorito roseo diventa livido, il respiro cessa. In questa morte apparente, perfino i polsi [...] immobili staran senza dar colpo $=$ no pulse shall keep his native progress, but surcease. Ogni parte del corpo, privata della sua capacità di muoversi (sì immota e fissa e d'ogni senso [...] priva = Each part depriv'd of supple government), sembrerà rigida (stiff), dura (stark) e fredda (cold).

Nel secondo e terzo atto, l'agg. Envious, che connota prima la luna (envious moon, act 2. sc. 2, v.3) e poi le strisce che colorano l'alba (envious streaks, act 3, sc. 5, v. 7), viene interpretato come 'malicious' da Gibbons ed Evans. Si tratta in realtà di un latinismo, col significato etimologico di 'full of envy, affected by envy' (Oxford Engl. Dict.) come dimostra il raffronto col Groto "O da invidia accelerata aurora »: nella percezione dell'amante, l'aurora arriva prima del tempo e questo suo affrettarsi è determinato dalla gelosia per la felicità dei due giovani. ${ }^{5}$

La scena cruciale dell'incontro notturno fra $i$ due amanti contiene - solo in Groto - il motivo dell'usignolo, che veglia insieme a loro (il rossignuol [...] con noi desto) e insieme a loro geme (con noi geme fra $\mathrm{i}$ spini). Shakespeare, nella sua ripresa, opera un tipico raddoppio, agganciando l'usignolo cantore della notte all'allodola annunciatrice dell'alba: si instaura cosi una ulteriore opposizione, che risponde alle altre coppie ossimoriche disseminate nel testo. ${ }^{52}$

Per l'allodola non è Groto la fonte, ma con ogni probabilità un passo di John Eliot, Ortho-epia Gallica, I593, p. I49: « Harke, harke, tis some other bird 
that sings now. / Tis a blacke-bird or a Nigtingale. / The Nightingale sings not but evening and morning. / Where is she I pray thee? / Tis a Nightingale I heard her record, / Seest thou not her sitting on a sprig? ".. 3 Fra le altre occorrenze dell'allodola nelle opere shakespeariane, il confronto più pertinente è con un passo di Venus and Adonis (pubblicato nello stesso anno I593, dunque anteriore alla stesura del Romeo and Juliet), cui rinvia per primo Knight: "Lo! here the gentle lark, weary of rest, / From his moist cabinet mounts up on high, / And wakes the morning, from whose silver breast / The sun ariseth in his majesty: / Who doth the world so gloriously behold / That cedar-tops and hills seem burnish'd gold» (cf. Second Ed., I864, in Furness I87I, cit., p. I9I).

Per completare l'analisi di questa scena, è opportuno richiamare l'attenzione sui vv. 24I-44 "Rivolgete la faccia a l'Oriente. / Ecco incomincia a spuntar l'alba fuori / Portando un altro Sol sopra la terra, / Che però dal mio Sol resterà vinto », corrispondenti in Shakespeare ai $v v$. 7-8 «Look, love, what envious streaks / Do lace the severing clouds in yonder east». Quanto al sintagma mio Sol, parallelo ad altro Sol, il rinvio è invece al primo dei passi citati, cioè all'inizio dell'episodio, quando il giovane amante attende nel giardino che l'innamorata appaia alla finestra. ${ }^{54}$

Rispetto ai luoghi paralleli indicati dalla critica shakespeariana, l'uno nel Labirinthus, ${ }^{5}$ l'altro nell'immancabile Brooke, ${ }^{56}$ appare evidente che la fonte grotiana si impone per la sua pertinenza, nella prospettiva anche di quella "light/dark imagery » che pervade l'intero dramma.

Tanto la donna-sole, quanto il sole a mezzanotte sono, d'altro lato, di pertinenza esclusiva della tradizione lirica italiana. L'identificazione metaforica della donna col sole risale agli stilnovisti (Guinizzelli ${ }^{57}$ e Cino da Pistoia), ${ }^{58}$ anche se la consacrazione definitiva del tema si deve a Petrarca, in quanto "l'immagine solare di Laura percorre l'intero Canzoniere »."

In modi sostanzialmente estranei all'archetipo del Petrarca ${ }^{60}$ si declina il tema nella tradizione cinquecentesca, ${ }^{61}$ che riprende l'equivalenza di base 'Donna = Sole' per sottoporla a variazioni inedite. ${ }^{62}$ Nel Groto lirico, Sole diviene né più né meno che il senhal della Donna amata, non identificabile con un referente biografico certo o comunque univoco. ${ }^{6}$

In quanto senhal, il vocabolo Sole percorre tutte le rime grotiane, ponendosi al centro di una irradiazione metaforica che pare trovare il proprio momento di sintesi in due testi nevralgici, la sestina $(I, 64)$, e la canzone Filosofia d'amor $(I, 83)$. Dell'uno e dell'altro si citano a titolo di esempio alcuni versi:

Ma quella invitta inespugnabil neve

Che 'ntorno smalta il cor del mio bel Sole,

Mai non allenta, anchor, che giunga il caldo 
Né l'amoroso, né l'estivo foco

Vince l'eterno inessorabil freddo

De la mia donna, e l'indomabil ghiaccio.

(I, 64, vv. 3I-36)

Non è ver, che a un bel Sole

Rigido gel si sfaccia,

Né che si liquefaccia

Bianca neve ove 'l fuoco giunger suole.

Che già strutta saria

A gli stessi occhi suoi la donna mia.

(I, 83, vv. 25-30)

$[\ldots]$

Non è ver, che velato

Il ciel d'oscura benda,

La vista altrui contenda.

$\mathrm{Al}$ maggior buio al'hor che è più serrato

L'aere, e che men riluce,

I' scorgo la mia bella, e chiara luce.

(ib., vv. 55-6o)

\section{$[\ldots]$}

Non è ver, che la vista

D'Amor la porta sia.

Ancho per altra via

La monarchia del cor cupido acquista.

Io pur di vista privo

Ardendo, amando, e desiando vivo.

(ib., vv. I०9-14)

Le coppie ossimoriche caldo/freddo, fuoco/ghiaccio, fiamma/neve, da Petrarca in poi indissolubilmente legate alla sintomatologia amorosa, sono dal Groto saldate all'altro elemento strutturante del proprio immaginario, l'opposizione fra tenebra e luce, notte e giorno, stelle (o luna) e Sole, fino a comprendere gli occhi, ora ciechi ora capaci di vedere. Ma anche di fronte al poeta privo della vista si impone, supremo ossimoro, ${ }^{64}$ l'immagine della Donna-Sole: gli occhi di lei sono una lucerna che illumina la notte a giorno, la sua figura fa impallidire le stelle, la sua presenza annulla l'oscurità, il volto di lei si imprime come un marchio a fuoco nel cuore del poeta.

Nella scelta - non banale - di questo senhal, possono aver interagito diverse sollecitazioni: il gusto per l'adynaton, sulla cui tradizione lirica è superfluo soffermarsi; ${ }^{65}$ l'esempio antitetico del Cariteo, che nel suo canzoniere traduce con Luna l'originario Selene del mito di Endimione, ${ }^{66}$ ivi celando il nome di 
una gentildonna, ${ }^{67}$ per poi intrecciarlo con le parole-rima giorno, notte, luce, sonno, e sole all'interno di una sestina (come in Groto, ma lungi dall'osservarne la perfetta bimembrazione ossimorica); ${ }^{68}$ infine, la Stella cantata dal Pontano negli Hendecasyllabi e ancor più negli Eridanorum libri, dedicati all'amore tardivo per la giovane ferrarese, il cui nome - non senhal - dà origine alla rete metaforica e al gioco di opposti che sono alla base di struggenti versi elegiaci. ${ }^{69}$

Nel Groto, il sole che spunta a mezzanotte come ipostasi della donna amata rinvia a un procedimento adynatico di cui si possono rintracciare esempi antichi, $^{70}$ ma che trova il proprio modello diretto in un passo delle Satire di Ludovico Ariosto:

$$
\begin{aligned}
& \text { Pazzo chi al suo signor contradir vole, } \\
& \text { se ben dicesse c'ha veduto il giorno } \\
& \text { pieno di stelle e a mezzanotte il sole. }{ }^{7 \text { I }}
\end{aligned}
$$

Legato al tema della follia in Ariosto, l'adynaton viene poi impiegato in ambito amoroso e lirico (non epistolare, come in Ariosto, né tragico, come in Groto) dal Cariteo, ${ }^{72}$ dal Boiardo, ${ }^{73}$ dall'Aquilano, ${ }^{74}$ dal Tebaldeo, ${ }^{75}$ da Battista Guarini (il Costante degli Eterei), ${ }^{76}$ fino agli ultimi e ormai stanchi echi del Marino. ${ }^{77}$

Il recupero di questa tessera ariostesca si spiega nel Groto con il desiderio di incrementare il ventaglio di metafore legate al senhal «Sole», senza con ciò dover attingere alla tradizione petrarchesca e petrarchista, da cui il Groto cerca di smarcarsi nel corso della sua traiettoria poetica. ${ }^{78}$ Di fatto, il Cieco è assai più sensibile alla produzione epigrammatica neolatina, come del resto dimostra la sezione compatta di epigrammi in volgare che occupa le cc. 222-300 della Prima Parte delle Rime, l'unica da lui curata in vita. ${ }^{79}$

Si è già detto del Pontano e della giovane Stella, che ne illuminò gli ultimi anni, ispirando parte degli Hendecasillabi e dell'Eridanus. Ma ancor più vicino al Groto, nello spirito e nella lettera, è un epigramma di Andrea Navagero, uno dei suoi elegantissimi Lusus:

\section{XXXVIII}

De Hyella

Nil tecum mihi iam Phoebe est, nil Nox mihi tecum,

A vobis non est noxve, diesve mihi.

Quantum ad me, ut libet auricomo Sol igneus axe

Exeat Eoae Tethyos e gremio,

Ut libet, inducat tacitas Nox atra tenebras:

Fert mihi noctem oculis, fert mihi Hyella diem. 




Nam quoties a me nitidos avertit ocellos, Ipsa in luce etiam nox tenebrosa premit. At quoties in me nitidos convertit ocellos, Candida et in media fit mihi nocte dies.

Non si tratta dunque di un modello latino classico, d'altronde inesistente, ${ }^{80}$ ma di un'immagine che si afferma agli inizi del Cinquecento con l'A riosto delle Satire $e$ gli epigrammi neolatini del Navagero, tra Ferrara e Venezia, nello spazio geografico e ideale dove il Cieco d'Adria trascorse la propria vita. Navagero riconduce alla cerchia aristocratica ed eterodossa di Domenico Venier, che fu amico e nume tutelare del Groto lirico. Ariosto marca invece un altro settore della produzione grotiana: a lui risalgono la predilezione per il capitolo in terza rima, ${ }^{81}$ un manipolo di epigrammi dedicati a personaggi del Furioso, ${ }^{82}$ l'ispirazione per le due commedie in endecasillabi sdruccioli, ${ }^{83}$ e una delle opere alimentari cui il Cieco collaborò a fianco di Gerolamo Ruscelli, ${ }^{84}$ cioè l'edizione commentata del poema, che uscì nel I5s6 con $i$ Cinque Canti in appendice, ${ }^{85}$ e gli argomenti in rima "di M. Luigi Grotta d'Adria ». ${ }^{86}$ Non sarà disutile riprodurre, a questo punto, due esempi dellidentificazione Donna $=$ Sole, tratti dalla Seconda $e$ Terza parte (normalmente ignorate) delle rime del Groto. ${ }^{87}$ Nel primo è distesamente proposta la similitudine fra la Donna e il Sole; nell'altro l'assenza della Donna provoca una serie di eventi (in)naturali che, dopo il consueto catalogo, si risolvono in un doppio Summationschema, figura dal Groto prediletta:

$$
\text { II, } \mathrm{I} 48
$$

Alla sua D. assimigliandola al Sole.

Tu del Sol vero illustre essempio rendi

Donna, in questo mortal nostro soggiorno,

Il Sol serena il Cielo, e accende il Giorno,

Tu sereni le menti, e i petti accendi.

Splende, ed abbaglia il Sole, tu abbagli, e splendi,

Arde egli in Granchio, e gela in Capricorno,

Tu foco, e giaccio al cor mio versi intorno,

Pioggie ei fà, tu a produr miei pianti intendi.

Il Sole indura il loto, il giaccio strugge,

Tu induri la mia fè, struggi il mio core,

Tu mi fai sfavillar, il Sol gli specchi.

Segue il Sol l'Elitropio, e l'Ombra fugge,

Te il mio cor segue e da te fugge Amore,

Le herbe e il sol, tu mia speme inverdi, e secchi. 


$$
\text { III, } 28
$$

Partita dalla sua Donna.

Di Madonna il partir non io sol sento,

Ma lo sente ogni tempo, ogni elemento,

Il giorno senza Sole, orbo, \& occulto

Giace nel mar sepolto.

La notte cieca, e bruna

Attende in van l'ottenebrata Luna,

Và senza stelle il Cielo,

L'aer senza seren veste altro velo,

L'acque de i fiumi senza pace al mare

Van tempestose, torbide, \& amare

La terra senza fiori a mezo Maggio

Mostra qual suole addur grandine oltraggio,

Rendi ad Hadria colei, che mi fà guerra,

E a Giorno, Notte, Cielo, Aere, Acqua, e Terra.

Fian resi senza indugio i sommi honori

Sol, Luna, Stelle, Seren, Pace, e Fiori.

La dispersione del tema lungo le oltre mille e trecento poesie, che costituiscono le tre parti delle Rime nell'edizione del I6I0, dispensa dal prolungare l'esemplificazione; ${ }^{88}$ vale la pena invece soffermarsi su una particolare variazione del motivo centrale, che collega la Donna/Sole all'alba o all'aurora, dunque necessariamente all'Oriente: III, 7 I Alla sua D. levata avanti giorno "Qual accidente si improviso, e presto / Lasciato l'opportuno almo soggiorno / A levar vi costringe avanti il giorno? / So no 'I fate per questo, / Che'l Mondo possa dir queste parole / - Stamane innanzi l'Alba è apparso il Sole »; III, 70 All'Aurora "Esca il tuo albor, più tosto che non suole, / Messaggiera di Febo, e ratto sgombra / La pena dal mio cor, dal mondo l'ombra. I E chiama col tuo raggio il tuo, il mio Sole »; III, 73 Occhi lodati, vv.I2-I4 "O Porte d'Oriente alme, e ben nate / Che chiuse a mezzo di date la notte, / E aperte a mezza notte, aprite il giorno ». Ma si veda soprattutto il distico finale del testo seguente:

$$
\begin{aligned}
& \text { III, } 69 \\
& \text { Udendo la sua donna. }
\end{aligned}
$$

Mentre con gli occhi volti all'Oriente

Contemplo il Sol nascente,

Che di rai luminosi coronato

Tutto superbo all'Orizonte ascende,

Ecco, che apparsa innanzi a me repente

L'alta mia Donna dal contrario lato, 
Tutta gioiosa splende.

All'hora il Sol, che si conobbe vinto

Dagli improvisi rai,

Di vermiglio color restò dipinto;

Et io scordato degli usati guai

D'alta felicità ciascun passai.

Ché spuntar vede un Sole sol, ciascuno,

Et io non ne veggio uno,

$\mathrm{Ma}$ in un tempo apparir duo Soli ho scorto

L'un dal Oceano uscir, l'altro dal Orto.

Di nuovo il motivo dell'alba, col sole che nasce sotto gli occhi dell'amante rivolti all'Oriente, la paronomasia Sole sol, la compresenza di duo Soli, l'opposizione Oceano/Orto.

Prendendo atto di questo retroterra culturale che lo precede e lo giustifica, acquista dunque nuovo spessore il verso e l'intera scena della Hadriana sopra citata (a. 2, sc. I, vv. 86-94): "Attenderò, che fuori esca Hadriana / [...] / A veder s'io ci son, com'è composto / Tra noi. E par, ch'io senta aprir la porta, / La qual meglio chiamar posso Oriente. ${ }^{89}$ / Ecco spunta il mio Sol cinto di nubi ${ }^{\circ}$ / A mezza notte » a cui fa eco, nel testo di Shakespeare, "But soft, what light through yonder window breaks? I It is the east and Juliet is the sun! ».

Barbara Spaggiari 
Il presente contributo riprende e sviluppa uno degli aspetti illustrati nella conferenza Luigi Groto: un poète provincial au rayonnement européen, presentata alla Faculté des Lettres dell'Università di Ginevra nel novembre 2008, su invito del Groupe d'Études sur les xvi ${ }^{\mathrm{e}}$ et $\mathrm{XVII}^{\mathrm{e}}$ siècles. Gli altri argomenti toccati in quella occasione, cioè l'influenza del Groto lirico sulle letterature iberiche (Camões, Quevedo e il barocco spagnolo), nonché le imitazioni delle sue opere drammatiche in ambito teatrale (da parte, fra gli altri, di Molière e di Georgios Chortatsis) saranno oggetto di ulteriori interventi. Chi scrive sta preparando l'edizione critica e commentata delle Rime di Luigi Groto, la cui ultima stampa risale al i6 Io.

I. Senza virgolette, o altri tentativi di mascherare, in omaggio a etichette alla moda, quello che resta un aspetto imprescindibile della ricerca e dell'analisi testuale.

2. Second series (1946-82), prima edizione, Methuen, 1980; poi Thomas Nelsons \& Sons, 1997 (reprint 2002). Si vedano anche il classico Romeo and Juliet. A New Variorum Edition of Shakespeare. Edited by Horace Howard Furness. New York 1963 [republication of the work first published by J. B. Lippincott \& Company in I 87I]; e il più recente Romeo and Juliet. Updated edition. Ed. by G. Blakemore Evans, Cambridge, Cambrigde University Press, I984 (2003), pp. 6-I 3 (dove in pratica il curatore nega qualunque influenza certa che non sia quella di Arthur Brooke).

3. A livello di archetipo, la «sfortunata morte di due infelicissimi amanti» (per dirla con Bandello) ha i suoi precedenti classici in Ovidio (Piramo e Tisbe, Met., IV 55-166; Ero e Leandro, Heroid. XIX: ambedue i miti sono puntualmente citati, l'uno dopo l'altro, dal Groto nel Prologo della Hadriana, rispettivamente ai vv. 63-69 e 70-74). Nel volgare italiano gli esempi si moltiplicano, dall'Istorietta amorosa fra Leonora de' Bardi e Ippolito Bondelmonti attribuita a Leon Battista Alberti (con l'anonimo rifacimento in ottave: «Incomincia il pietoso caso d'Ippolito e Dianora detto in rima »), all'altro poemetto in ottave di Gasparo Visconti, intitolato De dui amanti o De Paulo et Daria amanti (cfr. l'ed. di Beatrice Maltempi, tesi di dottorato, Urbino, A. A. 2000-200I), sino alle vicende di Iulia e Pruneo, narrate in una novella anonima della seconda metà del sec. XV (cfr. l'ed. dipl. di Luigi Monga, in «Esperienze Letterarie», XII, 1987, pp. 49-65). Quanto al topos letterario della 'morte apparente', due sono le sue principali linee di sviluppo: o l'evento imprevisto e accidentale (Boccaccio, Filoc. IV 67, ripreso in Decam. X, 4; il poemetto in ottave Storia di Ginevra degli Almieri; Matteo Bandello, Gerardo ed Elena), oppure lo stato di sonno profondo provocato intenzionalmente da un'apposita pozione (filtro magico, polvere, liquido distillato). A questa seconda tipologia appartengono le Ephesiaca di Senofonte di Efeso (sec. III d. C.); il Cligés di Chrétien de Troyes; la novella di Mariotto e Ganozza di Masuccio Salernitano (Novellino, XXXIII). Con esiti non tragici ma comici, sulla falsariga del Boccaccio, il motivo del beverone, o del sonnifero che simula la morte, è presente ancora in Giovanni Sercambi, Novelliere, Exemplo CLV (De pauco sentimento domini); Gentile Sermini, Novelle, I (Argomento: "Vannino da Perugia, innamorato della Montanina, donna d'Andreoccio [...] »); Giovanbattista Giraldi Cinzio, Hecatommithi, I 3, 5 (Argomento: «Consalvo, pigliata Agata per moglie, s'innamora di una meretrice [...]»). I testi corrispondenti, presentati in veste non sempre attendibile e suddivisi in sezioni secondo criteri talvolta discutibili, si possono leggere nella raccolta di Angelo Romano, Le storie di Giulietta e Romeo, 2 vol., Roma, Salerno, 1993 .

4. Sugli intermediari inglesi delle storie ispirate a Giulietta e Romeo, in italiano, francese o latino, cfr. René Pruvost, Matteo Bandello and Elizabethan Fiction, Paris, 1937 («Bibliothèque de la Révue de Littérature Comparée », t. I I 3 ) e Leo Salingar, Shakespeare and the Tradition of Comedy, London-New York, Cambridge Univ. Press, I974. Ė concordemente accettato il ruolo svolto da Painter col secondo libro di Palace of Pleasure, pubblicato nel 1567 (e ristampato una seconda volta, forse nel i 580 ), che contiene la versione inglese in prosa della novella di Matteo Bandello. 
5. Fra i precedenti inglesi, può considerarsi incontestata solo la traduzione in versi di Arthur Brooke, che si ispira anch'essa al testo del Bandello: cfr. The Tragical Historye of Romeus and Juliet written first in Italian by Bandell, and nowe in Englishe by Ar[thur] Br[ooke]. In aedibus Richardi Tottelli. Cum privilegio [1 562] (parzialmente riprodotta nell'ed. Gibbons, Appendix II: Extracts from Brooke's Romeus and Juliet, i 562 ).

6. Un esempio per tutti. La scena del commiato [episode], presente solo in parte della tradizione anteriore a Shakespeare, può aver luogo [(a) details]: (I) nella cella o nella chiesa di frate Lorenzo, (2) nel giardino di Giulietta, (3) sotto la sua finestra, (4) sotto il suo balcone. Romeo parla a Giulietta [(b) details]: (I) arrampicandosi su un muro, con o senza l'aiuto di una scala di corda; (2) attraverso una pesante inferriata; (3) dalla strada o da un casale di fronte; (4) direttamente nel giardino; (5) nella camera di lei dopo aver raggiunto il balcone.

7. Gibbons, cit., I980, p. 32.

8. Fu pubblicata per la prima volta dal Bendoni, in Venezia, poco dopo la morte dell'autore (avvenuta nel i 529 ), e ristampata dallo stesso tipografo nel i 535 . Per interessamento del fratello Bernardino Da Porto, una seconda redazione della novella, in larga parte rimaneggiata, fu data alle stampe dal Marcolini nel I 539, con il titolo La Giulietta. Le due versioni sono riprodotte a fronte da Marziano Guglielminetti, Novellieri del Cinquecento, MilanoNapoli, Ricciardi, I972, t. I, p. 24I e ss. Si noti che il veneziano Da Porto è il primo ad ambientare a Verona la storia dei due amanti infelici, pretendendo di riferirsi con ciò a fatti realmente accaduti; dopo di lui, nessuno metterà in discussione lo sfondo storicoambientale della vicenda, che rimarrà tenacemente ancorata al famoso - quanto fittizio balcone (come si sa, nessuna delle indagini finora svolte ha trovato il minimo supporto per autenticare i fatti).

9. Cfr. la dedica al Fracastoro. Composta verosimilmente durante il soggiorno veronese del Bandello (I 529 - I 536 ), la novella dal titolo La sfortunata morte di due infelicissimi amanti (II 9) fu stampata per la prima volta nel I 554, ancora vivo l'autore. Si veda ora Daria Perocco, La prima Giulietta: edirione critica e commentata delle novelle di Luigi Da Porto e Matteo Maria Bandello, Bari, Palomar, 2008.

Io. Questo nobile veronese pubblicò sotto pseudonimo L'infelice amore de i due fedelissimi amanti Giulia e Romeo scritto in ottava rima da Clizia nobile veronese ad Ardeo suo, Venezia, Giolito, I593, cioè un anno prima che apparisse la novella del Bandello (i due autori erano d'altronde in relazione diretta, come ha dimostrato il Brognoligo, La vita e le opere di Luigi Da Porto, in Id., Studi di storia letteraria,, Roma-Milano, Soc. Dante Alighieri, 1904, pp. 9- I 17). Il problema della priorità fra la novella e il poemetto non si pone, perché quest'ultimo è un chiaro rifacimento in versi della prosa bandelliana. La filiazione 'novella' > 'poemetto', 'prosa' > 'verso' è del resto una costante nella tradizione italiana della storia di Giulietta e Romeo (almeno quanto l'inverso è una costante della tradizione inglese).

I I. La princeps (Venezia, Farri, I 578) fu seguita da quattro ristampe cinquecentesche dei fratelli Zoppini (Venezia, I 582 , I 583 , I 5 86, I 599 ) e ancora da quattro edizioni veneziane nel primo quarto del Seicento (Sessa i6o9 e i6ro, Turrino i612, Spineda I626).

I 2. La prima tragedia, Dalida, composta da un Groto ancora adolescente, fu pubblicata nel I 572 (Venezia, Guerra) e rappresentata lo stesso anno sotto la Loggia del Palazzo Civico di Adria; seguirono sette ristampe, dal 1582 al 1646 , sempre in Venezia presso differenti editori (i fratelli Zoppini, Antonio Turrino, Gherardo Imberti, e lo Spineda). Delle commedie, tutte messe in scena almeno una volta ad Adria, la Calisto ebbe quattro edizioni, dal I 583 al I6 I 2, tutte presso i fratelli Zoppini, tranne l'ultima che uscì per i tipi del Turrino; l'Emilia cinque, dal i 579 al i6 i 2 (la princeps presso Francesco Ziletti, al quale subentrarono gli Zoppini; l'ultima uscí per i tipi del Turrino). Meno fortunate Il Tesoro (Venezia, Zoppini, I 583, I 586 e 1599; poi Turrino, I61 2) e La Alteria (Venezia, Zoppini, I 587; poi Turrino, 
I6 I 2). Il dramma sacro Isaac, rappresentato in Adria nel I 558 e I 58 I, uscì postumo presso gli Zoppini nel i s86, e ancora a Venezia nel i6os (a S.Angelo all'Insegna della Verità), nel i606 (per Gio. Battista Ciotti i606), nel i6 I 2 (per Antonio Turrino). Infine il Pentimento amoroso, dopo essere stato recitato in Adria nel is 65 e i 575 , ebbe due emissioni nel primo anno di stampa ( 576 : per Bolognino Zaltiero, e presso Francesco Rocca all'insegna del Castello) e altre sei edizioni veneziane dal i 583 al i6 i 2 (per gli Zoppini, i 583 , i 585 , e I 592 ; nel i6os a S.Angelo, all'insegna della Verità; nel r6o6, per Alessandro de' Vecchi; nel I6 I 2 , per Antonio Turrino).

I3. Cfr. la ricognizione di Luciana Zampolli, "Una scena di perpetua durevolezza »: le projet théatral de Luigi Groto, l'avengle d'Adria, in Robert Horville, Olinda Kleiman, Godeleine Logez, Théatre de cour, théâtre de ville, théatre de rue. Actes du Colloque International, 26-27-29 Novembre 1998, Lille, Université Lille 3, 2001, pp. 93-104. Si vedano in particolare le pp. I03-I04: «La renommée de l'œuvre théâtrale de Groto ne disparait pas après sa mort, mais il s'agit d'une renommée presque uniquement littéraire. Un exemple éloquent est le nombre considérable d'éditions posthumes de ses textes théâtraux (avec des écarts significatifs entre les différentes pièces). [...] Ce foisonnement éditorial n'a pas son correspondant dans l'activité théâtrale, ni à Adria ni ailleurs ». L'autrice attende alla stesura di una tesi di dottorato dal titolo L'activité théatrale de Luigi Groto, l'Avengle d'Adria, à la fin du XVI siècle, presso l’Université Paris-VIII.

I4. «In conclusion, the allusions to Daniel and Eliot indicate 1593 as the earliest possible date for Romeo and Juliet, the Bad Quarto makes 1596 the latest» (Gibbons, p. 3 I).

15. Volpone or the Fox è riconosciuta come la più brillante fra le commedie del drammaturgo inglese contemporaneo di Shakespeare e, all'epoca, a lui non inferiore per fama. Scritta in blank verse e ambientata a Venezia, la commedia annovera fra i personaggi una coppia di nobili inglesi, Sir Politic Would-be e la moglie, entrambi oggetto di pungente satira da parte dell'autore, l'uno per la patetica ossessione di essere vittima di un complotto, l'altra per le smanie di apparire à la page. Cfr. David C. McPherson, Shakespeare, Jonson, and the Myth of Venice, Cranbury NY, London and Mississauga Ont., Assoc. Univ. Presses, 1990, pp. 91-I I6; Michael J. Redmond, "I Have Read Them All». Jonson's 'Volpone' and the Discourse of the Italianate Englishman, in Michele Marrapodi (ed.), The Italian World of English Renaissance Drama. Cultural Exchange and Intertextuality, Univ. of Delaware Press, 1998, pp. I 22-40.

I6. Si cita dall'ed. di Michael Jamieson, London, Penguin, 2004 (1966); il commento a questo passo, p. 469, lascia non poco a desiderare: «Lady Would-Be correctly names the major Italian poets, including Giovanni Guarini (whose Pastor fido or Faithful Shepherd she is carrying with her), but Pietro Aretino, who wrote scurrilous and obscene satires, and Cieco di Hadria - 'the Blind Man of Hadria' - do not belong in this great company ». Il legame fra gli autori citati è piuttosto da ricercare nel genere letterario nel quale eccellono: la lirica per Petrarca e Dante (v. 93 "Your Petrarch is more passionate», v. 95 "Dante is hard, and few can understand him »); il romanzo cavalleresco per Ariosto e Tasso; la poesia drammatica per l'Aretino, il Guarini e il Groto.

17. Sulle competenze linguistiche di Ben Jonson e sul ruolo svolto da Giovanni Florio nella composizione del Volpone si vedano le pagine fondamentali di Mario Praz, L'Italia di Ben Jonson [1937], in Id., Machiavelli in Inghilterra e altri saggi sui rapporti letterari anglo-italiani, Firenze, Sansoni, 1962, pp. 195-210.

i 8. Francis Davison, $A$ Poetical Rapsodie, containing diuerse sonnets, odes, elegies, madrigals, epigrams, pastorals, eglogues, with other poems, both in rime and measured verse [...]. Newly corrected and augmented. Alla princeps seguirono ancora due edizioni: London, William Stansby for Roger Jackson, 16 I I (in $\mathrm{I}^{\circ}$ ) e London, B. Alsop for Roger Jackson, I62 I (in $8^{\circ}$ ). Nei due volumi dell'edizione curata da Hyder Edward Rollins (Harvard, Univ. 
Press, Mass., I931-1932) si tiene conto delle tre stampe antiche, I602-1621, riprodotte anche in facsimile.

I9. Con riferimento a Davison's Poetical Rhapsody, ed. by Arthur Henry Bullen, London, George Bell \& sons, 2 vol., I 890-1 891. Cfr. James Hutton, The Greek. Anthology in Italy to the Year I800, Ithaca-New York, Cornell Univ. Press, 1935, p. 328, nota 3.

20. L'edizione di riferimento è quella in due volumi a cura di Leon Emile Kastner (The Poetical Works of William Drummond of Hawthornden with «A Cypresse Grove», Edinburgh and London, William Blackwood and son, I913), non superata dalla successiva ed. di Robert H. McDonald, Poems and Prose, Edinburgh \& London, Scottish Academic Press, 1976.

21. D'altra parte, Drummond era un vero e proprio passeur, avendo tradotto, adattato o imitato autori non solo inglesi (Sidney, Shakespeare e Spencer), francesi (Ronsard e Pontus de Tyard), neolatini (Volpi, Zanchi, Castiglione), spagnoli (Boscán e Garcilaso), ma soprattutto italiani: Petrarca, Bembo, Sannazaro, Paterno, Guarini, Torquato Tasso, Marino e vari altri cinquecentisti. Cfr. Kastner, o.c., p. XLIII: «A full third of Drummond's compositions are translations or close paraphrases [...]. The rest are best described as adaptations from foreign models. Though the source of a small number of them has not yet been revealed, we may reasonably expect that one day the totality of his poems, with few exceptions, will be found to have been composed according to a given pattern, more or less vividly present in the poet's mind».

22. Segnalate per primo da Kastner, t. I, p. I67: «Sonnet vi, p. 6. Adapted from the following sonnet by Luigi Groto [Di produr perle Arabia non si vanti, in Rime, I 587, p. 65], a prolific author in various branches of literature, whose Rime (especially the madrigals), it may be noted in passing, were laid under heavy contribution by the brothers Davison in that part of Davisons' Poetical Rhapsody which appears under their names »; t. II, p. 333. «Sonnet xiv, p. I 3. Luigi Groto, who was on Drummond's shelves, has a sonnet on the same theme, beginning, Pelicano divin, da' col tuo sangue [in Rime, I 587, p. I77], which however has no resemblance in particulars with that of Drummond ».

23. Dal catalogo risultano, fra i libri italiani, la Commedia di Dante, il canzoniere del Petrarca e la Fiammetta del Boccaccio per il '300; poi il Furioso e le Rime dell'Ariosto; tutte le principali opere di Torquato Tasso; il Pastor fido del Guarini; le Rime di Sannazaro, Bembo, Della Casa, Cesare Caporali, Luigi Groto; le Nuove Fiamme di Ludovico Paterno; le raccolte di madrigali di Lelio Capilupi, Francesco Contarini, Muzio Manfredi, Cesare Rinaldi, Maurizio Moro, e Luigi Casone. Fra i cinquecentisti minori spicca la presenza del Bonardo, amico e protettore del Groto nella Fratta polesana, con i due discorsi Della miseria et eccellenza della vita bumana ( 5 86) dedicati a Lucrezia Gonzaga, e il trattato La minera del mondo (1585), di cui il Cieco fu il curatore. Cfr. LA | MiNerA | DEL MONDO, | Dell'Illustre S. ${ }^{R}$ Gio. Maria | Bonardo Frattegiano | Conte, \& Cavaliero; | Nella quale si tratta delle cose più secrete, e più rare de' $\mid$ corpi semplici nel mondo elementare, e de' corpi $\mid$ composti, inanimati, \& animati d'anima | vegetativa, sensitiva, e ragionevole. | Divisa in Quattro Libri, e per maggior commodità de' | Lettori ogni Capitolo, ch'ha potuto riceverlo, | ordinato, con ordine d'Alfabeto. | Mandata in luce, e dedicata da Luigi Grotto Cieco d'Hadria. | CON LICENTIA. | IN VENETIA, | Appresso Giacomo Zoppini, \& fratelli, I60o. Sul Bonardo e le sue opere si può consultare la monografia di Stefania Malavasi, Giovanni Maria Bonardo agronomo polesano del Cinquecento, Venezia, Deputazione di Storia Patria, 1988 (Miscellanea di Studi e Memorie, vol. XXV).

24. Cfr. The library of Drummond of Hawthornden, ed. by Robert H. MacDonald, Edinburgh, Edinburgh Univ. Press, 1971; il curatore la definisce un superbo esempio di biblioteca privata di un gentiluomo inglese all'inizio del sec. XVII.

25. Cfr. French Rowe Fogle, A Critical Study of William Drummond of Hawthorndon, New York, King's Crown Press, 1952, pp. 179-86. 
26. Nella sua indagine sulle fonti, Kastner ha però utilizzato l'ed. I 587 , che contiene solo la Prima Parte delle Rime: un'ulteriore ricerca che prendesse in conto la raccolta più completa riserverebbe, probabilmente, nuove sorprese sui debiti di Drummond nei confronti del Groto.

27. Com'è noto, il possesso di opere messe all'indice perché considerate di contenuto eretico fu alla base della condanna del Cieco da parte dell'Inquisizione nel i 567 . Nella lista dei libri 'eretici' requisiti in casa del Groto figurano appunto $i$ « Dialogi sette del reverendo padre frate Bernardino Occhino capuccino " (cfr. Giovanni Màntese - Mariano Nardello, Due processi per eresia. La vicenda religiosa di Luigi Groto, il "Cieco d'Adria", e della nobile vicentina Angelica Pigafetta-Piovene, Vicenza, Officine Grafiche, I974, alla p. 7I).

28. La questione è rapidamente liquidata dagli editori più recenti; cfr. Gibbons I 980: « Luigi Groto wrote a play in I 578 called Hadriana based on da Porto; it has a nightingale which sings when the lovers part, but it seems very unlikely that Shakespeare knew of it » (Introduction, p. 35); Evans 2003: «The proposed influence of Luigi Groto's La Hadriana ( 1578 ) and Lope de Vega's Castelveines y Monteses (not published until I647) is now generally discounted $»($ p. 7 , nota I).

29. Il più possibilista è Lloyd, Critical Essays, cit., che però suppone un intermediario inglese perduto: «The testimony of these extracts, all having great similarity from dependance on common authority, is, I think, not to be escaped from, that Sh. is here much closer to the italian drama [Hadriana] than to either of his English guides that remain. I therefore infer, on grounds already indicated, that he adapted or made use of some English adaptation of Groto, now lost» (cf. Furness i 87 I, cit., p. 404).

30. La Canace di Sperone Speroni (composta nel I 542, edita nel I 546) fu letta dall'autore davanti agli Accademici Infiammati di Padova. E si ricordi quanto afferma Trissino nella Quinta divisione della Poetica (ca. I 549, ma pubbl. postuma nel i 562 ): « la tragedia deve esistere di per sé, deve vivere nella e per la lettura, prima che nella e per la rappresentazione, deve essere dunque un sistema etico ed emozionale autosufficiente e scisso da ogni messinscena » (cfr. Siro Ferrone, Il teatro, Cap. XIII. \2. La tragedia: norme aristoteliche ed esperienze drammaturgiche. Trissino, Rucellai, Cinzio, Speroni, Aretino, Dolce, Tasso, in STOLI IV, 1996, pp. 909I009: alla p. 92I).

3I. Cfr. Giulietta Bazoli, Groto e Shakespeare: un confronto possibile?, in «Quaderni Veneti», 39 (2004), pp. 7-27, alla p. 17: «Durante le mie ricerche ho però trovato un riferimento esplicito al testo del Groto: l'Adriana infatti compare nell'elenco dei libri consultati da Giovanni Florio, insegnante di francese e italiano alle dipendenze del terzo conte di Southampton, Henry Wriothesley, per la compilazione della seconda ed. nel i6r i del Queen Anna's New World of Words $»$. Curioso, ma non troppo, che la stessa autrice presenti la propria ipotesi con scarso convincimento, come dimostra il punto interrogativo alla fine del titolo. Poco utile, anche se possibilista, il precedente articolo di Giancarlo Cavazzini, Dall" "Adriana » « Romeo and Juliet »: problemi di un rapporto, in Luigi Groto e il suo tempo. Atti del Convegno di studi (Adria, 27-29 aprile 1984), Rovigo, Minelliana, I987, t. I, pp. 337-53. Il riassunto della questione si ferma agli anni settanta del secolo passato (come del resto accade nell'altrimenti profittevole contributo della Bazoli). Presso i commentatori inglesi del testo shakespeariano, antichi e contemporanei, il problema viene accuratamente ignorato e le citazioni, che non oltrepassano la fine del sec. XIX, si limitano ai tre storici della letteratura che per primi affermarono l'esistenza di un legame fra l'Hadriana e Romeo and Juliet. Si tratta, com'è noto, di Joseph Cooper-Walker, Historical memoir on Italian Tragedy from the Earliest Period to the Present Time, London, for E.Harding, 1799 (poi Memoria storica sulla tragedia italiana di Giuseppe Cooper-Walker inglese. Versione italiana [di Bartolomeo Benincasa], Brescia, N. Bettoni, I81 г, pp. 56-59); Julius Leopold Klein, Geschichte des Dramas, I3 t. in I5 vol., Leipzig, T.O. Weigel, i 865-1 876: vol. IV (1866). Das Italienische Drama. I Bd., pp. 817-26 
Luigi Groto; vol.V (1867) Das Italienische Drama. II Bd., pp. 432-6r La Hadriana; infine William Watkiss Lloyd, Critical Essays on the Plays of Shakespeare, London: G. Bell and Sons, 1875. A favore dell'influenza grotiana si sono pronunciati anche John James Munro, Brooke's 'Romeus and Juliet', London, Chatto and Windus - New York, Duffield and Co., I 908, Introduction, pp. XxxvII ss.; e Olin H. Moore, The Legend of Romeo and Juliet, Columbus, The Ohio State Univ., I950, cap. XII Luigi Groto, pp. I03-Io. In Italia il più competente, convinto, ma anche rassegnato sostenitore di un legame fra la Hadriana e il Romeo and Juliet resta Mario Ariani, nelle pagine introduttive alla ristampa - con grafia modernizzata - della tragedia grotiana in Il teatro italiano. vol. II y La tragedia del Cinquecento, Torino, Einaudi, I977, pp. 28I-84 (il testo alle pp. $285-424$ ).

32. Cfr. David Orr, Italian Renaissance Drama in England before 1625. The Infuence of 'Erudita' Tragedy, Comedy, and Pastoral on Elizabethan and Jacobean Drama, Chapel Hill, Univ. of North Carolina Press, 1970, pp. ıо-ı г: «The only other ready sources for Italian books were the private libraries stocked with them. We know little about most of the libraries which might at time have held extensive Italian material, but we feel certain that that of John Florio contained Italian plays » (con rinvio a R. C. Simonini, Italian Scolarship in Renaissance England, Chapel Hill, Univ. of North Carolina, 1952, pp. I00-102 e I04 ss., riguardo a Florio e ai suoi rapporti, rispettivamente, con Shakespeare e Ben Jonson). Nel medesimo studio, Orr riserva una mezza paginetta dell'appendice alla questione "Shakespeare's Romeo and Juliet and Groto's La Hadriana » (p. I 27), osservando che «Of all attempts to prove a connection between Shakespeare and Italian tragedy, probably the most reasonable are those which attempt to show that Shakespeare used Groto's La Hadriana as at least a partial source for Romeo and Juliet. The only original italian tragedy to be brought to England was, as we have seen, Groto's La Dalida [p. 6r: «About I 592 a Latin play by William Alabaster was acted at Cambridge; it was a Senecan drama, but had as its definite and direct source the Italian play La Dalida, of Luigi Groto »]; and another play of his, a pastoral, Il Pentimento amoroso, also appears about this time. This is more evidence of appearence in England than we have for most Italian playwriters, but again it must be pointed out that both these Groto plays were translated in a university ». Rinvia quindi alla conclusione di Bullough (" very unlikely »), che dedica all'argomento meno di otto righe: «A play by Luigi Groto, Hadriana ( 1578 ) has a love affair drawn from da Porto in which a nightingale sings when the lovers part (Cp. RJ III.5.2), the opiate works as in Shakespeare, and the consolation offered to Capulet on his daughter's supposed death resembles Friar's Lawrence's in IV. $5.65 \mathrm{ff}$. It is very unlikely that Shakespeare knew this play. More directly in the line from da Porto to Shakespeare are a novella by Bandello and its French translation by Boiastuau [sic]» (cfr. Geoffrey Bullough, Narrative and Dramatic Sources of Shakespeare, t. I, London - New York, Routledge - Columbia Univ. Press, 1957, p. 271: il riferimento è alla traduzione di Pierre Boaistuau, Histoires tragiques extraictes des auvres italiennes de Bandel et mises en langue françoise, s.l., I 560 ). Sia l'approccio di Bullough che quello di Orr sono da considerare esemplari dell'atteggiamento al tempo stesso scettico e supercilioso che i critici anglosassoni manifestano verso possibili modelli italiani della tragedia elisabettiana, la cui importanza risulta in genere (non solo per Groto) minimizzata.

33. Com'è noto, nel Southampton è verosimilmente da riconoscere il giovane misterioso che ispirò la prima parte del book of sonnets.

34. Era nato nel 1593 da genitori italiani di confessione protestante, rifugiatisi in Inghilterra per sfuggire alle persecuzioni. Il padre Michelangelo, lui stesso tutor e teacher di italiano, fu autore fra l'altro di un manuale di Regole de la lingua thoscana (cfr. il testo edito, con introd. e note di Giuliano Pellegrini, in «Studi di Filologia Italiana», XII, 1954, pp. 77-204). John Florio svolse un'encomiabile opera per la diffusione della lingua e della cultura italiana fra gli intellettuali dell'epoca elisabettiana (Samuel Daniel, Ben Jonson, John Eliot, Thomas Nashe, Gabriel Harvey). Cominciò a Oxford come insegnante di italiano e di francese al Magdalen College; passò poi a Londra, dove si mantenne con lezioni private e lavorò per 
l'ambasciatore francese Michel de Castelnau; entrò finalmente al servizio del conte di Southampton nel i 589 e restò al suo fianco finché questi non cadde in disgrazia. Con l'avvento di Giacomo I si installò alla corte, dove svolse le funzioni di professore di lingue per il Principe di Galles, nonché di reader per la regina Anna. Numerosi gli elogi e i riconoscimenti in vita da parte di esponenti di spicco della cultura. Per Samuel Daniel, si veda qui la nota 37. Ben Jonson, nel dedicargli un esemplare di Volpone che si conserva nel British Museum, lo definisce «loving father and worthy friend»: cfr. Francis A. Yates, Italian teachers in Elizabethan England [1937-8], in Ead., Collected Essays, vol. II Renaissance and Reform: the Italian contribution, London, Warburg-Routledge, I983, pp. I64-80; ivi anche, pp. i6r-64, John Florio's Father [1955]. Alla Yates si devono ancora l'articolo John Florio at the French Embassy, in «Modern Language Review » 24 (1929), pp. i6-36, e la fondamentale monografia The Life of an Italian in Shakespeare's England, Cambridge, Cambridge Univ. Press, 1934. Anteriori, ma non disutili, i due studi di Clara Longworth de Chambrun, Shakespeare et Florio: études de vieux documents, Paris, La Revue, igr6; Ead., Giovanni Florio. Un apôtre de la Renaissance en Angleterre à l'époque de Shakespeare, Paris, Payot, I92 I, in particolare il cap. III, Les concordances, pp. 137-48, per i riscontri puntuali fra alcuni proverbi contenuti nell'opera di Florio e i versi corrispondenti dei drammi shakespeariani, ad es. "Florio Fast bind fast find (f.3 I): Merchant of Venise, II 5 Fast bind fast find, a proverb never stale in thrifty mind "; «Florio All that glistreth is not gold (f.32): Id., II 7 All that glitters is not gold, golden tombs do dust enfold ». Uno schizzo ironico e poco indulgente dei due Florio, padre e figlio, si può leggere in M. Praz, Giovanni Florio [1934], ora in Machiavelli in Inghilterra, cit., pp. I67-72. Sui possibili echi dei Fruits nelle opere shakespeariane si veda ora Keir Elam, 'At the cubiculo': Shakespeare's Problems with Italian Language and Culture, in Italian Culture in the Drama of Shakespeare and his Contemporaries: Rewriting, Remaking, Refashioning, ed. by Michele Marrapodi, Aldershot - Burlington, Ashgate, 2007, pp. 99-I Io (in particolare le pp. Iо0-102, e la nota 4$)$.

35. A Worlde of Wordes, or Most Copious and Exact Dictionnarie in Italian and English collected by John Florio, at London by Arnold Hatfield for Edward Blount, I 598, in-folio. La seconda edizione, considerevolmente aumentata (da circa 44.000 a oltre 70.000 entrate), fu redatta quando Florio era alle dipendenze di Anna di Danimarca, alla corte di Londra: cfr. Queen Anna's New World of Words collected and newly much angmented by John Florio, Reader of Italian unto the Sovereigne Maiestie of Anna, London, printed by Melchior Bradwood for Edw. Blount \& Wm. Berret. Anno I6ri (rist. anast. Menston, The Scholar Press, I968; vers. digitale disponibile su internet). Florio morì nel 1625 prima di aver completato la seconda parte della sua opera lessicografica, cioè il dizionario inglese-italiano, di cui restano alcuni fogli già composti.

36. Il dizionario bilingue costituiva solo un elemento nel progetto didattico, o meglio, culturale del Florio. Questo grande in-folio, stampato su tre colonne, contiene non soltanto liste di equivalenze fra vocaboli delle due lingue, ma anche espressioni e locuzioni più complesse, nonché citazioni tratte da opere letterarie, destinate a fornire modelli diretti di stile e di retorica ai letterati elisabettiani (la princeps uscì nello stesso anno dell'Euphues di John Lyly). Accanto al dizionario, due veri e propri manuali di conversazione, redatti dal Florio in forma di dialoghi, veicolano al tempo stesso aspetti della civiltà italiana e regole di buone maniere: i First Fruits (London, Thomas Dawon for Thomas Woodcoocke, I 578) dedicati a «familiar speech, merkle proverbes, wittie sentences and golden sayings. Also a perfect induction to the italian and english tongues »; i Second Fruits « to be gathered of twelve trees of diuerse but delightforme taftes to the tongues of Italians and Englishmen » (London, for Thomas Woodcoock dwelling at the Black Beare, I 59I) con in appendice "his Garden of recreation, yeelding six thousand Italian proverbes ». Sull'attività di John Florio si possono consultare Spartaco Gamberini, Lo studio dell'italiano in Inghilterra nel 's0o e nel ' 600 , Messina-Firenze, D'Anna, 1970; Enrico Borello, L'italiano come lingua straniera nel 'soo. John Florio e la glottodidattica, in "Quaderni del Dipartimento di Linguistica, Univ. degli Studi di Firenze », V (1994), pp. I 57-66; Alessandro Arcangeli, Les « Second Fruits» de John Florio ou la vie 
comme un jeu, in Shakespeare et le jeu. Actes du Congrès organisé par la Société Française Shakespeare (I0-I2 mars 2005), éd. par Yves Peyré et Pierre Kapitaniak, Paris, Univ. Paris-3, 2005, pp. I I-24. Non si dimentichi che Florio fu insegnante anche di francese, e per primo tradusse i dialoghi di Montaigne nella lingua di Shakespeare: alla sua opera di traduttore, piuttosto che alle opere originali per l'apprendimento dell'italiano, è d'altronde legata la sua fama postuma in Inghilterra. Esistono varie edizioni dei Montaigne's Essays translated by John Florio (London, I603), la più recente a cura di Graham Swift (London, Folio Society, 2006).

37. Tra cui quella di Samuel Daniel, To my deare friend and brother M. Iohn Florio, one of the Gentlemen of hir Maiesties Royall Priuy-chamber, vv.I-9: «I stand not to give praise before the face / Of this great worke, that doth it selfe commend: / But to congratulate the good and grace / That England com's thereby to apprehend: / And in hir name to thanke your industry / Laborious Florio, who have so much wrought / To honour hir in bringing Italy / To speake hir language, and to give hir note / Of all the treasure that rich tongue containes ».

38. Incisione di William Hole. Nell'ovale, a sinistra il motto « CHI SI CONTENTA GODE », a destra la data che conferma il I 593 come anno di nascita del Florio: «ÆT: 58. A. $^{\circ}$ D. ${ }^{1}$ I6II.». In basso, un epigramma latino concluso da una paronomasia: «In virtute sua contentus, nobilis arte, / Italus ore, Anglus pectore, uterque opere / Floret adhuc, et adhuc florebit; floreat ultra / FLORIVS, hac specie floridus, optat amans ».

39. L'accento che spesso figura a suggerire la corretta pronuncia delle parole risponde al proposito didattico delle opere di Florio, che non dimentica mai di essere, in primo luogo, un insegnante di lingue.

40. Per un nuovo approccio metodologico, basato sui concetti di « intertextuality », «interdiscursivity » $\mathrm{e}$ « interlexicality », che mira a rinnovare il «traditional method of comparative research ", si vedano le raccolte di saggi a cura di Michele Marrapodi - A.J. Hoenselaars (eds.), The Italian World of English Renaissance Drama: Cultural Exchange and Intertextuality, Newark, Univ. of Delaware Press, 1998; Shakespeare and intertextuality: the transition of cultures between Italy and England in the early modern period, ed. by M. Marrapodi, Roma, Bulzoni, 2000; e soprattutto Italian Culture in the Drama of Shakespeare and bis Contemporaries: Rewriting, Remaking, Refashioning, cit. alla nota 34.

4I. Sulla presunta limitatezza del bagaglio culturale di Shakespeare, e delle sue competenze in ambito linguistico, si veda la messa a punto di Robin Kirkpatrick, English and Italian Literature from Dante to Shakespeare. A Study of Source, Analogue and Divergence, London-New York, Longman, 1995 (ch. 8 Shakespeare and Italy, pp. 277-3 10). Concludono « in favour of a competent reading knowledge of Italian, via the mediation of Florio's work, on the part of Shakespeare and his fellow dramatists » le due recenti monografie di Jason Lawrence, Who the devil tought thee so much Italian? Italian Language Learning and Literary Imitation in Early Modern England, Manchester, Manchester Univ. Press, 2005, e Michael Wyatt, The Italian Encounter with Tudor England. A cultural Politics of Translation, Cambridge, Cambridge Univ. Press, 2005.

42. I testi sono tratti rispettivamente da LA | HADRIANA | TRAGEDIA | NOVA | DI LVTGI GROTO CIECO | D'HADriA. | Nuovamente ristampata. | IN VENETIA. | Appresso Fabio, \& Agostino Zopini Fratelli. | MDLXXXIII, pp. 54, 73-74 e 94-95; e dall'ed. Gibbons, cit., alle pp. I 27, I $84-85$ e $199-200$.

43. In Brooke, la scena si gioca tutta su elementi tratti dalla mitologia classica, Lucifero, Venere e Febo, senza alcuna identificazione della donna amata col sole: «But now (somewhat too soone) in farthest East arose / Fayre Lucifer, the golden starre that Lady Venus chose, / Whose course appoynted is, with spedy race to ronne, / A messenger of dawning daye, and of the rysing sonne. / Then freshe Aurora, with her pale and silver 


\section{Barbara Spaggiari}

glade, / Did clear the skyes, and from the earth, had chased ougly shade. / When thou ne lookest wide, ne closely dost thou winke, / When Phoebus from our hemysphere, in westerne wave doth sinke. / What cooller then the heavens do shew unto thine eyes, / The same, (or like) saw Romeus in farthest Esterne skyes / [...] / When golden crested Phoebus bosteth him in skye, / And under earth, to scape revenge, his dedly foe doth flye, / Then hath these lovers day an ende, their night begonne, / For eche of them to other is, as to the world the sunne» (vv. 1703-26: The lovers spend the night in the Juliet's bedchamber, in Arden Shakespeare, cit., p. 263).

44. Assente in Brooke.

45. Assente in Brooke.

46. Cfr. Brooke, vv. 2 I 49-57 (Juliet consults the Friar): « Receive this vyoll small, and keepe it as thine eye, / And on thy mariage day before the sunne doe clare the skye, / Fill it with water full, up to the very brim, / Then drinke it of, and thou shalt feele, throughout eche vayne and $\lim /$ A pleasant slumber slide, and quite dispred at length, / On all thy partes, from every part reve all thy kindly strength. / Withouten moving thus thy ydle parts shall rest, / No pulse shall goe, ne hart once beate within thy hollow brest, / But thou shalt lye as she that dyeth in a traunce» (Arden Shakespeare, cit., pp. 266-67).

47. Sulla stessa linea l'autocommento di Dante a Vita Nova, I6.I 4 (son. Spesse frate vegnonmi a la mente, vv. I 2-I 4 " e se io levo li occhi per guardare, / nel cor mi si comincia uno tremoto, / che fa de' polsi l'anima partire): «La terza si è che quando questa battaglia d'Amore mi pugnava così, io mi movea quasi discolorito tutto per vedere questa donna, credendo che mi difendesse la sua veduta da questa battaglia [...]. La quarta si è come cotale veduta non solamente non mi difendea, ma finalmente disconfiggea la mia poca vita ».

48. Il solo sintagma "vene e polsi » figura ancora in SACCHETTI, Il Libro delle Rime, I69, I03 (ed. F. Brambilla Ageno, Firenze, Olschki, r990) e Angelo Galu, Canzoniere, 259, 479 e 270, I I (ed. G. Nonni, Urbino, Accademia Raffaello, I987); le riprese più complete in DrAgonetto Bonifacio, Rime, 48, 49 «Onde, per polsi e vene mi scorre un gelo allor tutto tremante» (ed. R. Girardi, Fasano, Schena, i 995) e Benedetto Varchi, Rime, i, 533, 7 «Sentir quaggiù, le vene tutte e i polsi / Tremar d'ardente giel non dubbio segno » (in Opere, Milano, Treves, I 858-1859).

49. «intanto che la bevanda dell'oppio facendo suo corso, le vene e i polsi vennero a risserrarsi, e in poco d'ora spirò ».

50. Cfr. Gasparo Visconti, De dui amanti, ottava 26 (e ultima del poemetto, giunto incompleto): «Costui cercando dove il polso batta / sol per intender s'ha de vita dramma, / un poco a Daria di calore accatta / che sotto move a la sinistra mamma, / e disse: - Morte ancor non ha disfatta / a questo corpo la vital sua fiamma, / e spero sana farla in spazio breve, / ché questo non è caso molto greve ». Le parole sono di un medico chiamato da Paulo per visitare il corpo esanime di Daria.

SI. In contesto non erotico, si ricordi $O$ lente, lente currite noctis equi di Christopher Marlowe (cfr. The Tragical History of Doctor Faustus, Act 5, Scene 2, v. Io), splendido adattamento di un pentametro tratto dagli ovidiani Amorum lib. I, xIII, 40. In pieno Cinquecento, il motivo si innesta nel reperto paremiologico festina lente, immortalato negli Adagia di Erasmo e visualizzato, ad esempio, nel noto emblema di Aldo Manuzio, un’àncora secca che rallenta il nuoto del delfino.

52. Sull'antitesi in generale, e sulla «light/dark imagery» nel dramma shakespeariano, cfr. Gibbons, cit., Introduction, $\ 4$ (in particolare le pp. I8-19). 
53. Rinvio segnalato per primo da J. W. Lever, Shakespeare's French fruits, in «Shakespeare Survey », 6 (1953), pp. 82-83.

54. Il balcone è un'innovazione di Q2 (= Second Quarto, I 599, usato come copy-text da tutti gli editori moderni); cfr. la nota di Gibbons, ad loc., « According to Qi [First Quarto, o 'bad' Quarto, I 597] Juliet appears at a window in the tiring-house façade, not at a balcony ».

55. Cfr. Francis Douce, Illustrations of Shakespeare, London, Longman, i 807: «This line in particular, and perhaps the whole scene, has been imitated by the author of the Latin Comedy of Labirinthus. In Act III, iv, two lovers meet at night, and the Romeo of the piece says to his mistress, 'Quid mihi noctem commemoras, mea salus? Splendens nunc subito illuxit dies, ubi tu primum, mea lux, oculorum radiis hasce dispulisti tenebras' » (in Furness I 87 I, cit., p. 94).

56. Cfr. Evans, nota p. Io6 «Juliet is the sun. Compare Brooke (1726) 'For eche of them to other is, as to the world, the sunne'».

57. Guido Guinizzelli, Rime, I, 2 I-23 « Ben si pò tener alta quanto vòle, / ché la plu bella donna è che si trove / ed infra l'altre par lucente sole / e falle disparer a tutte prove ». Per il tema della donna-luce in Guinizzelli cfr. Furio Brugnolo, 'Parabola' di un sonetto del Guinizzelli, in AA.VV., Per Guido Guinizzelli, Padova, Antenore, 1980, pp. 53-105 (alla p. 68).

58. Cino da Pistoia 5, 9-i 2 «Ancor m'ha fatto Amor più ricco dono, / ch'a tal donna m'ha dato in potestate, / che vede 'l sole, là dov'ella appare, / cosa che 'l vince di sua chiaritate».

59. Santagata, nota a $R v f$. 4, I 2 « ed or di picciol borgo un sol n'a dato »; ivi anche i rinvii a tutti i luoghi relativi dei $R v f$, tra cui si segnalano per la loro rilevanza ai nostri fini $R v f$. 90 , I 2-I 3 « uno spirto celeste, un vivo sole / fu quel ch'i’ vidi »; Rvf. Iо०, I-2 «Quella fenestra ove l'un sol si vede, / quando a lui piace, et l'altro in su la nona »; Rvf. 175, 9 "Quel sol, che solo agli occhi mei resplende » (con paronomasia sol / solo); Rvf. 219, 9- I 4 " Così mi sveglio a salutar l'aurora, / e 'l sol ch'è seco, et più l'altro ond'io fui / ne' primi anni abagliato, et son anchora. / I' gli ò veduti alcun giorno ambedui » (con attacco di tipo epigrammatico, il cui modello è reperibile in Cic., De nat. deorum, I 28, 79, cfr. Santagata, ad loc.); Rvf. 225, I-2 «Dodici donne honestamente lasse, / anzi dodici stelle, e 'n mezzo un sole» (da cfr. con TC III I 33 e I 25-26); Rvf. 226, 3-4 « ch'io non veggio 'l bel viso, et non conosco / altro sol, né quest'occhi ànn'altro obiecto »; Rvf. 248, 2-4 "costei, / ch'è sola un sol, non pur a li occhi mei, / ma al mondo cieco, che vertù non cura » (di nuovo la paronomasia sola / sol, per cui Santagata ad loc. suggerisce un possibile rinvio alla paretimologia di Isidoro, Etym. 8, I I 53 《solem dixerunt, quasi solum»); Rvf. 255, 4-6 «la matina è per me più felice hora: / ché spesso in un momento apron allora / l'un sole e l'altro quasi duo levanti »; Rvf. 275, I-2 « Occhi miei, oscurato è 'l vostro sole; / anzi è salito al cielo et ivi splende » (oltre ai modelli biblici, cfr. Bernart de Ventadorn Ara no vei luzir solelh / tan me son escurzit li rai, segnalato da Maurizio Perugi, Petrarca provenzale, in «Quaderni Petrarcheschi», VII, I990, pp. I09-8I, alla p. 172); Rvf. 338, I "Lasciato ài, Morte, senza sole il mondo » (per cui cfr. anche $R v f$. 246, Io e $R v f$. 352, I 2-1 3); $R v f$. 363, I « Morte à spento quel sol ch'abagliar suolmi » (per cui cfr. $R v f$. 231, 8 « che 'l sol de la mia vita à quasi spento »).

6o. In Petrarca, oltre all'intreccio del tema solare con il mito dafneo, che si evidenzia a partire da $R v f$. 5, 3, è marcante l'identificazione del Sole con Cristo, secondo un procedimento tipico della tradizione cristologica (e non mariana; v. infra, nota 62). Cfr. Fredi Chiappelli, Le thème de la 'Defectio solis' dans le 'Canzoniere': 'variatio intus', in Mélanges Rycbner, Strasbourg, 1978, pp. 75-84 (poi in F. Chiappelli, Il legame musaico, a c. di P. M. Forni e G. Cavallini, Roma, Ed. di Storia e Letteratura, I984, pp. 165-79); e sulla solaris virtus $\mathrm{cfr}$. Vincenzo Fera, I sonetti CLXXXVIe CLXXXVII, in « Lecturae Petrarcae », VII (1987), pp. 219-43 (alla p. 226 e nota 30). 
6r. Secondo un numero limitato di schemi ripetitivi: vedere la Donna è vedere il Sole; soltanto agli occhi del poeta è dato vedere la Donna come un Sole; la vista della donna abbaglia gli occhi del poeta; la morte della donna priva il poeta (e il mondo) del suo Sole. Lo scarto adynatico interviene in senso opposto rispetto al Groto: non il sole a mezzanotte, semmai un'eclissi, cioè l'assenza del sole in pieno giorno.

62. Nell'evidenza relativa alla lirica del Cinquecento non sarà da escludere, fra l'altro, il recupero diretto della fonte biblica, Quasi sol refulgens (Eccli 50, 7-8), che già l'esegesi patristica si era precocemente incaricata di deviare dal referente originale - Simone, figlio di Onia, exemplum di vir iustus - per applicarla alla Vergine. A partire da una certa altezza, la doppia metafora dell'Ecclesiastico (Quasi sol refulgens [...] et quasi arcus refulgens inter nebulas) entra di diritto a far parte delle omelie «In Die Festo Sanctae Mariae», legandosi in particolare all'Assunzione. Non sembra invece imporsi, almeno nella lirica, la problematica di origine cabalistica relativa alla coppia 'sole'/luna', per la quale si rimanda al numero unico, intitolato Il sole e la luna, della rivista "Micrologus », I 2 (2004) e alla relativa bibliografia.

63. È del resto una costante del Groto mescolare i due piani della realtà e della convenzione letteraria: per prova, le numerose allusioni alla propria cecità che si affiancano a versi in cui, parlando in prima persona, il poeta si attribuisce una facoltà visiva, di cui in realtà era privo fino dalla nascita.

64. Il riferimento è d'obbligo alla teoria dell'amore che passa per gli occhi, sintetizzata nel famoso passo di Andrea Cappellano, De Amore, lib. I, 5, 6 « Caecitas impedit amorem, quia caecus videre non potest» (cfr. ed. E. Trojel, Copenhague, I 892; rist. München, I972; e con trad. ted. a fronte, W. De Gruyter, 2006).

65. Per rimanere nello stesso ambito semantico, si vedano ad es. Petrarca, Rvf. 237, i6-i 8 «Ben fia, prima ch'i' posi, il mar senz'onde, / et la sua luce avrà 'l sol da la luna, / e i fior' d'april morranno in ogni piaggia »; FILenIo GaLlo, Rime, II 98, 73 «Pria da la luna arà sua luce el sole, / Maggio senza viole... » (ed. M. A. Grignani, Firenze, Olschi, 1973); Torquato TAsso, Rime, 37, 8 «o luna che ne faccia il sole oscuro» (ed. B. Maier, Milano, Rizzoli, 1963-1964).

66. Cfr. Le rime di Benedetto Gareth detto il Chariteo secondo le due stampe originali, con introd. e note di E. Pèrcopo, II, Napoli, r 892, p. 8. Il Pèrcopo pubblica l'Endimione secondo la stampa del i 509 (Napoli, Sigismundo Mayr). Sul Cariteo si vedano Giovanni Parenti, Benet Garret detto il Cariteo. Profilo di un poeta, Firenze, Olschki, I993; Beatrice Barbiellini Amidei, Alla luna. Saggio sulla poesia del Cariteo, Firenze, La Nuova Italia Editrice, 1999; e cfr. anche Marco Santagata, La lirica aragonese. Studi sulla poesia napoletana del secondo Quattrocento, Padova, Antenore, 1979, pp. 296-34I.

67. Appartenente alle famiglie Chiaramonte o Montalto. L'Endimione fu edito per la prima volta nel i 506, e tre anni dopo nella forma definitiva.

68. Cfr. Sestina I, Quel ch'io no' spero mai vedere il giorno. Alle coppie antitetiche giorno/notte, sole/luna si affianca la correlazione asimmetrica luce $\sim$ sonno, che trova il proprio corrispettivo non in rima, ma all'interno del verso: cfr. v. 3 « veggio dormendo ne la oscura notte », v. Io «ch'io resto oscuro, quando gli altri han luce », v. I I «tanto riposo, quanto dura il sonno », e l'intera quarta strofe: «Quando comincia uscir quell'altra luna / Dal nostro mar, per dar lume a la notte, / Allhor m'addormo et veggio l'alma luce / Di quella, che mi scalda più che 'l sole; / Ma, poi che aprendo gli occhi, vedo il giorno, / Conosco che mia gloria è ombra et sonno ».

69. Cfr. ad es. Hendec. I 28, I0-1 8 «Sed iam plus solito nitescit aër, / iam lux candidior diem serenat: / cur ah, cur tenebrae repente nobis, / cur nox exoritur, nigrescit aura? / An sentis, miser, an miselle, sentis? / Stella est ad speculam, refulsit in te: / solem lumina victa 
pertimescunt. / O claras medio die tenebras, / o lucem sine nube nigricantem »; Eridan. VIII De Stella «Nostra die quod Stella nitet, quod nocte refulget, / solem Stella die, sidera nocte refert. / Nocte eadem surgente nitet, cedente refulget, / Phosphoron hic, illic Hesperon ipsa refert. / Ergo eadem mihi sol, eadem mihi sidus et una / Lucifer est, eadem Vesper et una mihi »; X Ad Stellam « In tenebris, mea Stella, nites, dum sidera pallent, / et nox ipsa tuo lucida honore placet; / in medio, mea Stella, die sub sole nitescis, / clarior et per te solque diesque venit. / [...] / Sed cur, quae tenebris honor es, decus una diebus, / cur lumen nobis nocte dieque negas? / Nocte negas foribus clausis, in luce fenestris: / o tenebrae, non iam stellave luxve mihi »; De Stella XXVII, $5-8$ « Stella mihi sol est, eadem mihi roscida luna; / hinc fovet et flammis, irrigat hinc et aquis; / Stella eadem ferit ex oculis oculisque medetur; / haec mihi et hostis Amor, haec et amica Venus ».

70. Da Apuleio, Metam. XI, 23 «nocte media vidi solem candido coruscantem lumine » (nel viaggio agli Inferi che integra i riti iniziatici dei cultori di Iside), a Petrarca, Rime disperse e attribuite, I4, 5 «Farebbe a mezza notte ardere il sole, / E primavera quando è maggior verno» (detto di Madonna).

71. Sat. I, ı-1 2. Com'è noto, la prima satira è indirizzata dall'Ariosto al fratello minore Alessandro e all'amico Ludovico da Bagno che hanno seguíto il cardinale Ippolito in Ungheria, nella sua nuova sede vescovile di Agria (Eger). Non si deve contraddire il proprio signore, dice il poeta, anche se afferma come verità vissuta una serie di impossibilia.

72. In almeno due occasioni, il sonetto 39 dell'Endimione, vv. 5-6 « [Donna] Possete fare a mezza notte il giorno / Col tenero, suave e bianco petto »; e Altre rime, I4, 6 «Non vidi tu la fiamma nel mio core, / Che mostra ad meza nocte un chiaro lume?».

73. Cfr. Amorum Libri, 3, I 3 (son. Tanto son peregrine al mondo e nove) in cui Giove « né tanto se ralegra aver adorno / il ciel di stelle, e aver creato il sole / che gira al mondo splendido d'intorno, / quanto creato aver costei, che sole / scoprir di terra a meza notte un giorno / e ornar di rose il verno e di viole».

74. Capitolo 7, Dell' Aurora, vv. I- I 2: « Ben poi tu lucidar, candida Aurora, / Con la tua vista el mondo e 'l cielo intorno, / Ché le tenebre mie cominciano hora, / Rompendo a me col tuo crudel ritorno / Spesse volte un tal sogno e sì suave, / Che a meza nocte a me fa lieto il giorno. / Ché dove Amor di me pietà non have, / Havea sognando in braccio el mio bel sole / Al tuo dispecto et di soe voglie prave ». Cfr. anche «Lucido fai con lei ogni contorno / Talhor non vai di tenebre vestito, / Ché a meza nocte fai parer il giorno » (Strambotti, Capitolo 6, 63).

75. Rime, 30I, 2 «Ove è il bel sol che cum sua luce chiara / te facea giorno a meza nocte oscura, / nuda fenestra, che già tanto dura / me fusti a torto, e sì sdegnosa e avara? ».

76. Rime degli Accademici Eterei, Padova, i 567, 34, 4 « Vedovo e fosco albergo, almo soggiorno / di lei, che 'n te suol far chiaro oriente, / e voi cieche contrade ove sovente / scoprir già vidi a meza notte il giorno, / dopo un lungo silenzio ecco a voi torno » (ed. G. Auzzas - M. Pastore Stocchi, Padova, Cedem, I995).

77. Cfr. La Sampogna, II, 430-32 « onde parea / spuntar ne l'antro oscuro / a meza notte l'alba e, lampeggiando » » (ed. V. De Maldé, Parma, Guanda, 1993).

78. Il riconoscimento di una presunta forma-canzoniere soggiacente all'organizzazione delle Rime di Luigi Groto non regge ad una lettura obiettiva, anche volendosi limitare alla Prima Parte, come hanno sempre fatto sinora i pochi studiosi che si sono occupati della produzione lirica del Cieco d'Adria. Cfr. Alessio Togni, La formula dell'equivoco nella poesia di Luigi Groto il Cieco d'Adria, memoria di licenza, Università di Friburgo - Svizzera, 1982 (in particolare, le pp. 46-47 e i 46-66). 
79. Autore egli stesso di poesia neolatina, Groto volle chiudere il proprio libro di rime con i Carmina da lui stesso composti, trentuno in totale, nei quali si rinvengono temi, stilemi e figure retoriche che in nulla si differenziano rispetto ai componimenti volgari. Solo a partire dalle edizioni postume (nella fattispecie, I $587 \mathrm{~B}$ ), il volumetto della Prima Parte delle Rime fu decurtato della sezione finale e così ristampato fino al i6ro, sopprimendo di fatto una componente essenziale della personalità dell'autore, che i pochi studiosi moderni hanno fin qui accuratamente ignorato. Nell'anno is 87 uscirono, di fatto, dalla tipografia degli Zoppini due edizioni (non emissioni) delle Rime del Groto, da noi siglate i $587 \mathrm{~A}$ e i $587 \mathrm{~B}$, soltanto la prima delle quali è corredata dei Carmina latina; oltre a questa differenza fondamentale, le due edizioni del i 587 offrono altre varianti sostanziali che permettono di identificare i due rami della tradizione a stampa ad esse successiva.

8o. Come già segnalato sopra a proposito del Petrarca, l'immagine del Sole era legata in origine alla cristologia. A ulteriore prova, l'unica occorrenza reperibile in tutta la tradizione della poesia latina, dall'antichità classica al medioevo, è un verso di Alexander Neckam, De laudibus divinae sapientiae, 5, $95 \mathrm{I}$ "Tunc sol in terris media de nocte refulsit», riferito appunto alla nascita del Cristo. Non basta: questo panegirico, scritto pochi anni prima della morte (ca. I 2 I 3), non è altro che il rifacimento in versi del trattato De naturis rerum, opera in prosa a carattere enciclopedico che lo stesso Neckam compose prima del i 205. I due elementi, come dimostra Jean-Yves Tilliette, rilevano della pratica dell'opus geminum, in cui «les deux réalisations de la même œuvre ne diffèrent que par la forme, le poème étant alors conçu comme la pure et simple paraphrase versifiée du traité en prose » (J.-Y. Tilliette, Rhétorique de l'encyclopédie: le cas du De naturis rerum d'Alexander Neckam (vers I200), in La transmission des savoirs au Moyen Âge et à la Renaissance. Vol. I: $D u X I I^{e}$ au $X V^{e}$ siècle, sous la direction de Pierre Nobel. Actes du Colloque [...], Tours, 24-29 mars 2003, Presse Univ. Franche-Comté, 2005, pp. 289-302: alla p. 29I). Sull'autore e le sue opere resta fondamentale la monografia di Richard W. Hunt, The Schools and the Cloister. The Life and Writings of Alexander Nequam (II57-I2I7), edited and revised by Margaret Gibson, Oxford, Clarendon, I984: della tradizione manoscritta della Laus divine sapientie si parla alle pp. I 23-24 e i 38; l'unica edizione a stampa è infatti ottocentesca: cfr. Thomas Wright, Alexandri Neckam «De naturis rerum libri duo» with "De laudibus divinae sapientiae distinctiones decem», Rolls Series, London, I863, pp. 357-503 (in realtà le parti dell'opera, cioè le distinctiones, non sono dieci ma sette, come provato già da Hunt, cit., p. I38; e cfr. Tilliette, cit., p. 29I, nota 8). Dunque anche quest'unico specimine del sole a mezzanotte nella latinità medievale è in realtà la trasposizione in versi di un'immagine che trova la propria collocazione nei commenti patristici e nelle opere esegetiche legate alla tradizione cristiana del topos.

8 . Rara nella Prima Parte delle Rime, la terza rima occupa un posto di tutto rilievo nell'edizione del i6ro.

82. Cfr. 225 Ruggier, poich' hebbe liberato Angelica, 226 Angelica, 238 Marfisa con due briglie in mano, 239 Bradamante sopra il padre, e'l marito morti. Si cita dalla princeps: LA PRIMA PARTE | DELLE RIME | Di Luigi Groto Cieco di Hadria. | Con privilegro. | [ritratto del Groto] | Venetia, Per Fabio, e Agostin Zopini fratelli. | M.D.LXXVII, alle cc. I 32-37 (nostra la numerazione delle poesie).

83. Come La Lena e Il Negromante, anche L'Emilia e L'Alteria del Groto (la prima rappresentata nel i 579, la seconda con dedica al Bonardo datata i 584 ) utilizzano, oltre al metro ariostesco, spunti del Boccaccio su uno sfondo genericamente plautino. Si veda l'allusione al personaggio dell'Ariosto che compare, in guisa d'ammiccamento, nel Prologo dell'Alteria: «Vi farò brevemente il tutto intendere. / Son Nigromante, e dalle solitudini / Dell'Egitto co i libri, che Pier d'Abano / Lasciò morendo, son qui giunto a istantia / De l'auttor, che vi forma ogni anno favole / Sceniche, per poi farvene spettacolo: / Che già vi diè l'Hadriana, la Dalida, / Il Pentimento Amoroso, l'Emilia, / La Calisto, e 'l Thesoro: c'hor l'Alteria / Vuol darvi che così vuol, che si nomini / Questa fatta da lui nova 
Comedia ». Si cita dall'ed. del I587, c. $7 \mathrm{v}$ (cfr. LA Alteria | COMEDIA | NOva | DI LVIGI | grotto | CIECO D'HADRLA. | Dedicata al molto Illustre Sig. Conte, e | Caualiere Il Sig. Gio. Maria | Bonardo Fratteggiano. | Nuouamente posta in luce. | IN VeNETIA MdcLXxxviI | Appresso Fabio, \& Agostino Zoppini Fratelli).

84. L'altra è il cosiddetto Decamerone riformato, per cui cfr. IL | DECAMERONE | DI MESSER | GIOVANNI BOCCACCIO | CitTAdin FIORENTINo. $\mid$ Di nuovo riformato da $\mid$ LUIGI groto CIECO D'ADRIA | Con permisione de' Superiori. Et con le Dicbiarationi \& Avvertimenti, di Girolamo Ruscelli. | cON PRIVILEgI. | IN VENETIA, MDXC | Appresso Fabio, \& Agostino Zoppini Fratelli, | \& Onofrio Farri compagni. Sulla sua vicenda tormentata (dall'incarico ricevuto per sostituire il testo della Crusca, fino allo smarrimento del manoscritto negli anfratti della censura romana) non è ora il caso di soffermarsi. Si veda intanto Jacqueline Tschiesche, Il rifacimento del Decamerone di Luigi Groto, in Atti Adria, cit., 1987, t. I, pp. 237-69, e G. Chiecchi - L. Troisio, La riforma del Groto, in AA.VV., Il Decamerone sequestrato. Le tre edizioni censurate nel cinquecento, Milano, Unicopli, I984, pp. 86-104.

85. Non ci risulta che quest'appendice abbia fatto oggetto di indagine per ciò che è degli elementi testuali, e ci riserviamo di trattare la questione in altra sede. Per i Cinque Canti si rinvia intanto all'edizione e al commento di Cesare Segre (Lodovico Ariosto, Opere minori, Milano-Napoli, Ricciardi, I954, pp. 58 I-82), da integrare con Segre, Studi sui "Cinque canti", in «Studi di filologia italiana», XII, I954, pp. 23-75; sulle successive prese di posizione si veda Alberto Casadei, Il percorso del «Furioso ». Ricerche intorno alle redazioni del I5I6 e del IS2I, Bologna, il Mulino, I 993, pp. I I 3-27 e 175-92, che per suo conto conclude in favore di una giunta al Furioso la cui prima stesura risalirebbe agli anni i 5 I9-I 52 I (collocandosi dunque fra la redazione A e B). L'ipotesi è accolta da Eugenio Refini, L'isola-balena tra «Furioso» $e$ "Cinque Canti", in «Italianistica », XXXVII, 3 (2008), pp. 87-I I I (ivi anche tutta la bibliografia relativa alla questione, p. 88, nota I). Da segnalare infine Vincenzo Monforte, L'attività di Luigi Groto in margine ai "Cinque Canti» dell' Ariosto, in Atti Adria, cit., I987, t. I, pp. $273-88$.

86. Grotta per Groto (o Grotto) figura in tutte le edizioni, cfr. I CINQVE | CANTI | DI M. Lodovico | ARIOSTO, | I quali seguono la materia del Furioso. | Tutti di nuovo revisti, \& ricorretti da molti importantissimi | errori, che fin qui sono stati in tutti gli altri. | CON GLI ARGOMENTI IN RIMA, | \& discorsi di M. Luigi Grotta d'Adria. | CON ALCUNE BREVI ET IMPORTANTI | Annotationi del medesimo. | IN VENETIA | Appresso Nicolò Moretti. Il nome non figura invece nel frontespizio dell'ed. da noi consultata: ORLANDO FVRIOSO | DI M. LODOvico | ARiosto, | Tutto ricorretto, \& di nuove Figure adornato, | Con le Annotationi, gli Avvertimenti, \& le Dichiarationi di Ieronimo Ruscelli. | La Vita dell'Autore descritta dal Signor Giovanbattista Pigna. | Gli Scontri de' luoghi mutati dall'Autore dopo la prima impressione. | La dichiaratione di tutte le Istorie, \& Favole toccate nel presente libro. | fatte da M. Nicolò Eugenico. | DI nuovo Aggiuntovi | Li Cinque Canti del medesimo Autore. | Et una Tavola de' principij di tutte le Stanze, | Con altre cose utili, \& necessarie. | CON PRIVILEgIO. | IN VENETIA, M.DC.III. | Appresso Felice Valgrisi.

87. Cf. RIME | di Lvigi Groto | Cieco d'Hadria. | Parte Prima. | A cui seguono altre due | Parti hora di novo | date in luce. | Con la vita dell'Autore. | Et con la Tavola delli | argomenti. | Al molto Illustre et Eccl. Sig. | Strozzi Cicogna Vicentino. | Con privilegro. | IN Venetia | Appresso Ambrosio Dei | igio.

88. Si va dai versi isolati, che presuppongono l'identificazione come dato di fatto (cfr. ad es. II, 39 Lingue, cori, \& occhi bramati, v. 3 « Per mirar meglio voi mio chiaro sole »; III, I I 4 Alle nubi, vv. 8-9 « E come l'ombra vostra via se 'n vole / Il suo la terra, ed io vedrò il mio Sole "), alle variazioni sul tema mediante una serie di opposita: notte/sole (cfr. ad es. III, i 56 Epitafio dell'Autore, vv. 22-25 «Sperai goder la luce, / E notte eterna a gli occhi miei si induce. / Né lo star chiuso in tal notte mi duole, / Ma che tal notte nasce dal mio Sole»; III, 52 , Alla ditta nel ballo, vv. 9-I4 «La pietà vostra qualitati estreme / Onde ancor meraviglia il cor 


\section{Barbara Spaggiari}

m'ingombra / Ciel terra, Altezza, \& humiltade unio. / Luce, Tenebra, Sole \& ombra insieme, / Voi Cielo, io Terra, alta voi humile io, / Luce voi, Tenebra io, voi Sole, io Ombra »), oppure sole/Sole = astro/Donna (cfr. ad es. III, 5 s Per un ballo, vv. I-8 «Mentre con lieto salto / Quasi real Augel ti levi in alto, / Mira, che così in alto non ti levi, / Che alla sfera del Sole, ò al foco ascenda, / Onde il tuo crin si accenda, / E del viso si struggano le nevi. / Che 'l Mondo all'hor diria queste parole, / Nel foco il foco, e nel Sol arde il Sole »).

89. In altro contesto, ma con la stessa volontà definitoria, il verso è probabilmente un'eco di Par. XI, 5 2- 54 « Però chi d'esso loco fa parole, / non dica Ascesi, ché direbbe corto, / ma Orïente, se proprio dir vuole »; cf. la nota corrispondente di Anna Maria Chiavacci Leonardi: «se vi è nato il sole, si dovrà chiamarlo Oriente, se si vuol essere proprio esatti » (Dante Alighieri, Commedia, vol. III Paradiso, Milano, Mondadori, i997, p. 3 I4).

90. Cfr. act 3, sc. 5, vv. 7-8 « what envious streaks / Do lace the severing clouds in yonder east ». 